\title{
SMOOTH $S^{1}$ ACTIONS ON HOMOTOPY COMPLEX PROJECTIVE SPACES AND RELATED TOPICS ${ }^{1}$
}

\author{
BY TED PETRIE
}

This paper is dedicated to Professors Leroy M. Kelly and Fritz Herzog who gave so enthusiastically of their time and talent in developing undergraduate mathematicians at Michigan State University. I was one of their beneficiaries.

0 . Introduction and motivation. We begin by listing some questions and remarks which establish the theme of this paper.

1. Which cobordism classes of oriented manifolds admit nontrivial circle actions? Answer: Atiyah-Hirzebruch [4]: For a compact oriented manifold $X$ of $\operatorname{dim} 4 k$, its $\hat{\mathscr{A}}$ genus vanishes iff there is a multiple $m X$ which is cobordant to $Y$, with $W_{2}(Y)=0$, which admits a nontrivial circle action on each of its components. The $\mathscr{A}$ genus is the genus belonging to the power series $(x / 2)(\sinh x / 2)^{-1}$.

2. Which manifolds in a given homotopy type admit nontrivial circle actions? More specifically, of those manifolds homotopy equivalent to complex projective $n$ space, which admit nontrivial $S^{1}$ actions?

Strong conjecture. If $h: X \rightarrow C P^{n}$ is an orientation preserving homotopy equivalence and if $X$ supports a nontrivial circle action then $h^{*} \mathscr{A}\left(C P^{n}\right)$ $=\hat{A}(X)$ where

$$
\hat{\mathscr{A}}(X)=\prod\left(x_{i} / 2\right)\left(\sinh x_{i} / 2\right)^{-1} \in H^{*}(X, Q)
$$

and the elementary symmetric functions of the $x_{i}^{2}$ give the Pontrjagin classes of $X$. In other words, the homotopy equivalence must preserve the total $\mathscr{A}$ cohomology class.

Weak conjecture. To the hypothesis of the strong conjecture add the condition that the fixed point set of the action consists of isolated fixed points. Then

$$
h^{*} \hat{\mathscr{A}}\left(C P^{n}\right)=\hat{\mathscr{A}}(X)
$$

A corollary of the strong conjecture is that most homotopy complex projective spaces do not admit $S^{1}$ actions. The weak conjecture is discussed in detail in Part II, $\$ 2$.

An invited address delivered to the 684th Meeting of the Society in New York, on April 10, 1971; received by the editors August 19, 1971 .

AMS 1970 subject classifications. Primary 57D65, 57E05; Secondary 20C10.

Key words and phrases. Equivariant $K$ theory, nonsingular bilinear forms, equivariant Poincaré duality, $\operatorname{spin}^{c}$ bundles, Atiyah-Singer Index Theorem, representation on normal fibers, equivariant line bundles.

${ }^{1}$ Partially supported by an NSF Grant. 
The validity of the weak conjecture is related to the representations of $S^{1}$ on the tangent space of $X$ at the isolated fixed points. If $X$ is homotopy equivalent to $C P^{n}$, there must be $n+1$ isolated fixed points $p_{j}$. To each we show how to associate an integer $a_{j}$ and compare the eigenvalues of the $S^{1}$ action on the tangent space of $X$ at $p_{j}$ with the integers $\left\{ \pm\left(a_{k}-a_{j}\right)\right.$, $k \neq j\}$.

A particularly good property of the homotopy type which is useful to exploit in connection with the second question is the existence of a $\operatorname{spin}^{c}$ structure. In the first few sections we discuss the properties of an equivariant $\operatorname{spin}^{c}$ structure.

Another idea we develop in connection with $S^{1}$ actions on manifolds in general, is the exploitation of a theorem of Stewart (Part I, 6.1) which is concerned with lifting an $S^{1}$ action on $X$ to an $S^{1}$ action on a principle $S^{1}$ bundle over $X$. Using this theorem and assuming $H^{1}(X, Z)=0$, we define a function $F$ from the additive group $H^{2}(X, Z)$ to the multiplicative group of units of $K_{S^{1}}^{*}(X)$. Assuming $X$ is a spin ${ }^{c}$ manifold and using Stewart's theorem we construct an "orientation class" $\delta_{S^{1}} \in K_{S^{1}}^{*}(T X)(T X=$ tangent bundle of $X)$. This class generates $K_{S^{1}}^{*}(T X)$ as a free module over $K_{S^{1}}^{*}(X)$.

The index homomorphism $\operatorname{Id}_{S^{1}}^{X}: K_{S^{1}}^{*}(T X) \rightarrow R\left(S^{1}\right)$ is a homomorphism of $R\left(S^{1}\right)$ modules and is intimately connected to the representations of $S^{1}$ on the normal fibers of the components of the fixed point set. Suppose that $z_{1}, \ldots, z_{s}$ is a basis for $H^{2}(X, Z)$ and let $\Phi\left(y_{1}, \ldots, y_{s}\right)$ be any polynomial in indeterminants $y_{i}$ with integer coefficients. Set $w_{i}=F\left(z_{i}\right) \in K_{S^{1}}^{*}(X)$. Then the condition that

$$
\operatorname{Id}_{S^{1}}^{X}\left(\delta_{S^{1}} \Phi\left(w_{1}, w_{2}, \ldots, w_{S}\right)\right) \in R\left(S^{1}\right)
$$

for every $\Phi$ imposes stringent restrictions on the representations of $S^{1}$ on the normal fibers of the components of the fixed point set. This idea is exploited in connection with Part II, Theorems 2.11 and 2.12.

The principle applications of the ideas developed here are in Part II, Theorems 2.8-2.12. They deal with the relationship between $\mathscr{A}(X)$, the integers $\left\{\left(a_{k}-a_{j}\right)\right\}$ and the integers $\left\{x_{j k}\right\}$ which are the roots of the $S^{1}$ action on $T X$ at $p_{j}$.

Another interesting item, which was suggested by the above mentioned results, is an example of an exotic action of $S^{1}$ on $C P^{3}$. It is exotic in the sense that the eigenvalues of the $S^{1}$ action on $T C P^{3}$ at the four isolated fixed points are distinct from those of the linear case (Part I, 6.4). See Part II, $\S 4$ for more detail. Another significant feature of this example is the fact that the bilinear form $\langle>$ of Part II, $\$ 3$ is nondegenerate in this case, see $\S 5$ of Part II.

We have interspersed the ideas and theorems with numerous examples and conjectures. We hope the reader finds the former of sufficient interest to consider the latter. 
This paper is divided into two parts and is organized as follows:

I. GENERALITIES CONCERNING SMOOTH ACTIONS OF COMPACT LIE GROUPS ON MANIFOLDS

1. Properties of the index homomorphism $\operatorname{Id}_{G}^{X}: K_{G}^{*}(T X) \rightarrow R(G)$.

2. The group $\operatorname{spin}^{c}(m)$.

(a) The half spin representations $\Delta_{+}$and $\Delta_{-}$as complex $\operatorname{spin}^{c}(m)$ modules.

(b) The elliptic pairing of $\operatorname{spin}^{c}(m)$ modules:

$$
R^{m} \times \Delta_{ \pm} \rightarrow \Delta_{\mp} .
$$

3. $\operatorname{Spin}^{c}(m)$ bundles.

4. $K_{G}$ orientation of $G$ manifolds and Poincaré duality.

(a) Equivariant homology dual to $K_{G}^{*}$.

(b) Examples of orientations constructed from equivariant $\operatorname{spin}^{c}(m)$ structures.

5. Formula for $\operatorname{Id}_{G}^{X}: K_{G}(T X) \rightarrow R(G)$ in terms of:

(a) Orientation class of $X$.

(b) Representations of $G$ on normal fibers to fixed point set.

6. Specialization to $S^{1}$ actions.

(a) Stewart's theorem.

(b) The homomorphism from $H^{2}(X, Z)$ to the group of units of $K_{S^{1}}^{*}(X)$.

(c) Standard example-Illustration of (a) and (b) for the case of "linear actions" of $S^{1}$ on $C P^{n}$.

\section{Applications to $S^{1}$ ACtions ON A HOMOtopy COMPLEX PROJECTION SPACE $X$ AND SPECULATIONS}

\section{Generalities.}

(a) The equivariant "Hopf bundle" $\eta \in K_{S^{1}}^{*}(X)$.

(b) The integers $a_{j}$ associated to the component $X_{j}$ of the fixed point set of the $S^{1}$ action by restricting $\eta$ to a point $p_{j} \in X_{j}$.

(c) Comparison of $K_{S^{1}}^{*}(X)$ with $K_{S^{1}}^{*}\left(X^{Z_{p} r}\right), X^{Z_{p^{r}}}=$ fixed point set of $Z_{p^{r}} \subset S^{1}$.

2. $S^{1}$ actions on $X$ with isolated fixed points.

(a) Number theoretic properties of the eigenvalues of the representations of $S^{1}$ on the tangent space at the isolated fixed points.

(b) Theorem 2.8; The relations between the eigenvalues of the representations of $S^{1}$ above and the integers $a_{j}$ defined by the equivariant Hopf bundle $\eta$.

(c) The class $\hat{\mathscr{A}}(X) \in H^{*}(X, Q)$. 
3. Speculation: The bilinear form $\left\langle>\right.$ on $K_{G}^{*}(X)$.

(a) Analogy with cup product pairing for ordinary cohomology theory.

(b) When is $\langle>$ nondegenerate over $R(G)$ ?

(c) Examples where \langle\rangle is nondegenerate.

4. An exotic action of $S^{1}$ on $C P^{3}$.

(a) Exotic representations on $T C P^{3}$ at isolated fixed points.

(b) Identification of differential structure.

5. The bilinear form $\left\langle>\right.$ on $K_{S^{1}}^{*}(X), X=C P^{3}$.

It is indeed a pleasure to acknowledge my gratitude to Glen Bredon who made several important suggestions concerning the material of this paper. Also, one should consult the work of W. Y. Hsiang referenced in the bibliography for related ideas.

\section{GeNeRALITIES CONCERNING SMOOTH ACTIONS OF COMPACT LIE GROUPS ON MANIFOLDS}

1. Properties of the index homomorphism. $\operatorname{Id}_{G}^{X}: K_{G}^{*}(T X) \rightarrow R(G)$. Here we review the relevant properties of the equivariant $K$ theory of [1], [5] and [6]. Throughout, $G$ is a compact Lie group acting smoothly on a manifold $X$. Denote by $K_{G}^{*}(X)$ the equivariant $K$ theory of $X$. We note that $K_{G}^{*}(Y)$ is defined for any locally compact $G$ space $Y$, in particular for $Y=T X$ the tangent space of $X$. In this case, $K_{G}^{*}(T X)$ is a module over $K_{G}^{*}(X)$ via $\pi^{*}$ where $\pi: T X \rightarrow X$ is the projection.

If $i: Z \rightarrow X$ is the inclusion of a $G$ invariant submanifold $Z$ whose normal bundle $v^{\prime}$ is complex, there is a homomorphism

$$
i_{*}: K_{G}^{*}(Z) \rightarrow K_{G}^{*}(X)
$$

with the property

$$
i^{*} i_{*}(x)=\lambda_{-1}\left(v^{\prime}\right) \cdot x
$$

when $x \in K_{G}^{*}(Z)$ and $\lambda_{-1}: K_{G}^{*}(Z) \rightarrow K_{G}^{*}(Z)$ is the operation which sends a $G$ vector bundle $\xi$ to $\sum(-1)^{i} \lambda^{i}(\xi), \lambda^{i}(\xi)$ is the $i$ th exterior power of $\xi$.

We note that $T Z \subset T X$ always has a complex normal bundle namely $\pi^{*}(v \otimes C)$ where $\pi: T Z \rightarrow Z$ is the projection and $v$ is the normal bundle of $Z$ in $X$. Thus if $T i$ denotes the inclusion of $T Z$ in $T X$, the homomorphism

$$
T i_{*}: K_{G}^{*}(T Z) \rightarrow K_{G}^{*}(T X)
$$

satisfies

$$
T i^{*} T i_{*} x=\lambda_{-1}(v \otimes C) \cdot X
$$

for $x \in K_{G}^{*}(T Z)$. Recall $K_{G}^{*}(T Z)$ is a $K_{G}^{*}(X)$ module via $\pi^{*}$. 
Note that $K_{G}$ (point) $=R(G)$ is the complex representation ring of $G$ and $K_{G}^{*}(X)$ is an $R(G)$ module. An important example is the case $G=S^{1}$, the circle group. Then $R\left(S^{1}\right)=Z\left[t, t^{-1}\right]$ is the ring of Laurent series $\sum_{i=-N}^{N} a_{i} t^{i}$. Here $N$ is an arbitrary positive integer and all $a_{i}$ are integers.

Of fundamental importance is the existence of a homomorphism of $R(G)$ modules:

$$
\operatorname{Id}_{G}^{X}: K_{G}^{*}(T X) \rightarrow K_{G}^{*}(\mathrm{pt})=R(G) ;
$$

$R(G)$ is the complex representation ring of $G$ (character ring of $G$ ). This homomorphism satisfies a few basic properties which makes it quite accessible to computation. Let $\omega: G^{1} \rightarrow G$ be a homomorphism. Then there is a homomorphism $\omega^{*}: K_{G}^{*}(T X) \rightarrow K_{G}^{*}(T X)$ and a commutative diagram

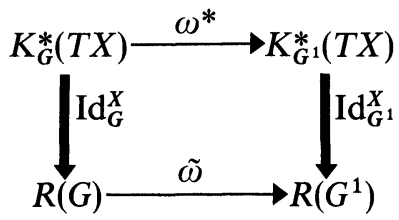

(Compatibility axiom).

Of course $\omega^{*}$ is defined for any $G$ space $Y$. If $i: Z \rightarrow X$ is the inclusion of an invariant submanifold, then there is a commutative diagram

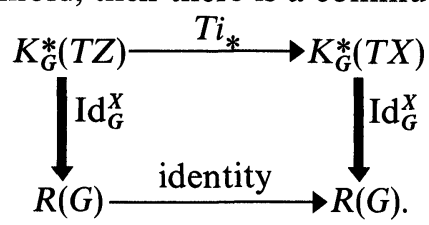

(1.5) If $X$ is a point, $\operatorname{Id}_{X}^{G}$ is the identity map of $R(G)=K_{G}^{*}(T X)$.

Let $G$ be abelian and $g \in G$. Denote by $\mathfrak{p}$ the prime ideal of characters of $R(G)$ which vanish at $g$. The localized ring $R(G)_{p}$ consists of the fractions $\{\chi / \psi \mid \chi, \psi \in R(G), \psi(g) \neq 0\}$ with the relation $\chi_{1} / \psi_{1}=\chi_{2} / \psi_{2}$ if there is an $\omega \in R(G)$ with $\omega(g) \neq 0$ and $\omega\left(\chi_{1} \psi_{2}-\chi_{2} \psi_{1}\right)=0$. If $M$ is an $R(G)$ module $M_{\mathfrak{p}}=M \otimes_{R(G)} R(G)_{\mathfrak{p}}$.

If $S$ is a subset of $G, X^{S}$ denotes the set of points of $X$ fixed by elements of $S$. Note that since $G$ is abelian, $X^{g}$ is a $G$ invariant submanifold of $X$ for $g \in G$. There is then this basic theorem of Atiyah-Segal [5].

(1.6) Localization Theorem. The inclusion $i: X^{g} \rightarrow X$ induces isomorphisms $i_{\mathfrak{p}}^{*}: K_{G}^{*}(X)_{\mathfrak{p}} \rightarrow K_{G}^{*}\left(X^{q}\right)_{\mathfrak{p}}$ and $T i_{\mathfrak{p}}^{*}: K_{G}^{*}(T X)_{\mathfrak{p}} \rightarrow K_{G}^{*}\left(T X^{g}\right)_{\mathfrak{p}}$. The latter has inverse $\lambda_{-1}(v \otimes C)^{-1}(T i)_{p^{*}}$ where $v$ is the normal bundle of $X^{g}$ in $X$.

Thus $\operatorname{Id}_{G}^{X}$ is completely determined by (1.4), (1.5) and (1.6) in the case $X^{g}$ consists of isolated points. 
When $v$ is a $G$ vector bundle over $X$ and $Z \subset X$ is an invariant submanifold, we denote by $\left.v\right|_{Z}$ this bundle restricted to $Z$. If $Z=x$ is a fixed point, it is a complex $G$ module and we let $\left.v\right|_{x}(g)$ denote the trace of the element $g$ acting on $v_{x}$ for $g \in G$, i.e., the value at $g$ of the character of $G$ defined by $v_{x}$.

2. The group $\operatorname{spin}^{c}(m)$. Let $V$ be a real vector space of dimension $m=2 n$. We suppose $V$ endowed with the standard inner product with respect to an orthonormal base $e_{1}, e_{2}, \ldots, e_{m}$. Let $A(V)$ denote the Clifford algebra of $V$ [2], [13]. For $v \in V \subset A(V)$ we have

$$
v^{2}=-\|v\|^{2} \cdot 1
$$

where $1 \in A(V)$ is the identity.

$A(V)$ is the direct sum $A^{+} \oplus A^{-}$where $A^{+}$is spanned by the products $e_{i_{1}} e_{i_{2}} \cdots e_{i_{k}}$ with $k$ even and $A^{-}$by the products with odd $k$. The multiplicative subgroup of $A(V)$ generated by elements of the unit sphere $S^{m-1} \subset V$ $\subset A(V)$ is denoted by $\operatorname{spin}(m)$. The intersection $\operatorname{spin}(m) \cap A^{+}$is the group $\operatorname{spin}(m)$.

The group $\operatorname{spin}(m)$ acts in an obvious manner on $A^{+} \otimes C$ giving a linear representation of $\operatorname{spin}(m)$. This representation is reducible

$$
A^{+} \otimes C=2^{m}\left(\Delta_{+} \oplus \Delta_{-}\right)
$$

where $\Delta_{+}$is the + eigenspace of $(i)^{n} e_{1} e_{2} \cdots e_{m}=\tau$ and $\Delta_{-}$is the negative eigenspace of $\tau$.

Observe that $\tau^{2}=1$ and $\tau$ commutes with elements of $A^{+}$and so with $\operatorname{spin}(m)$ and

$$
\tau v=-v \tau \text { for } v \in V .
$$

Because of this, left multiplication by $v \in V$, denoted by $L(v)$, maps $\Delta_{+}$to $\Delta_{-}$and vice versa. Let

$$
\theta: V \times \Delta_{ \pm} \rightarrow V \times \Delta_{\mp}
$$

be the map defined by

$$
\theta(v, \delta)=(v, L(v) \delta), \quad v \in V, \delta \in \Delta_{ \pm} .
$$

Then $\theta$ is elliptic, i.e., for fixed $v \neq 0$ in $V$ the linear map

$$
\theta_{v}: v \times \Delta_{ \pm} \rightarrow v \times \Delta_{\mp}
$$

defined by restricting $\theta$ is an isomorphism. This follows from the fact that

$$
\theta_{v} \circ \theta_{v}(v, \delta)=(v, L(v) L(v) \delta)=\left(v,-\|v\|^{2} \delta\right)
$$

because $L(v) \circ L(v)=L\left(v^{2}\right)=-\|v\|^{2} \cdot 1$ by $(2.1)$. 
The generator $\varepsilon=-1 \in A(v)$ of the double covering

$$
\pi_{1}: \operatorname{spin}(m) \rightarrow S O(m)
$$

acts as multiplication by -1 on $\Delta_{+}$and $\Delta_{-}$. This means that the action of $\operatorname{spin}(m)$ on these two representation spaces may be extended to the group

$$
\operatorname{spin}^{c}(m)=\operatorname{spin}(m) \times_{Z_{2}} S^{1} \cdot{ }^{2}
$$

Here $Z_{2} \subset \operatorname{spin}(m)$ is the subgroup generated by $-1 \in \operatorname{spin}(m)$ and $Z_{2} \subset S^{1}$ is the subgroup generated by $-1 \subset S^{1}$. Explicitly if $[g, t]$ denotes an equivalence class in $\operatorname{spin}^{c}(m)$ determined by $g \in \operatorname{spin}(m)$ and $t \in S^{1} \in C$, then

$$
[g, t] \delta=t \cdot(g \cdot \delta) \text { for } \delta \in \Delta_{ \pm} .
$$

Of particular importance to us is the commutative diagram

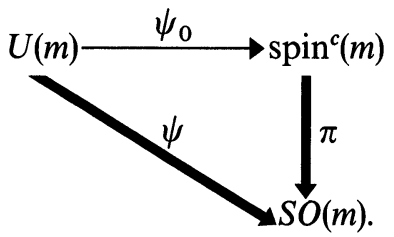

Here $\pi[g, t]=\pi_{1}(g)$

$$
\psi \operatorname{diag}\left(e^{i \theta_{1}}, e^{i \theta_{2}}, \ldots, e^{i \theta_{n}}\right)=\operatorname{diag}\left(\begin{array}{cc}
\cos \theta_{j} & \sin \theta_{j} \\
-\sin \theta_{j} & \cos \theta_{j}
\end{array}\right) \subset S O(m),
$$

$\psi_{0} \operatorname{diag}\left(e^{i \theta_{1}}, e^{i \theta^{2}}, \ldots, e^{i \theta_{n}}\right)$

$$
=\left[\prod_{j=1}^{n}\left(\cos \theta_{j} / 2-\sin \theta_{j} / 2 e_{2 j-1} e_{2 j}\right), \exp \left[-i\left(\sum \theta_{j} / 2\right)\right]\right] \text {. }
$$

Note that

$$
\prod_{j=1}^{n}\left(\cos \theta_{j} / 2-\sin \theta_{j} / 2 e_{2 j-1} e_{2 j}\right) \in \operatorname{spin}(m) \subset A(V)
$$

so $\psi_{0}$ makes sense and $\pi \psi_{0}=\psi$.

Observe that $\operatorname{spin}^{c}(m)$ has a central circle subgroup $S^{1}$ and the quotient is $S O(m)$. The orbit map is $\pi$.

Moreover there is an exact sequence of groups

$$
1 \rightarrow \operatorname{spin}(m) \stackrel{i}{\rightarrow} \operatorname{spin}^{c}(m) \stackrel{j}{\rightarrow} S^{1} \rightarrow 1, \quad j[g, t]=t^{2},
$$

\footnotetext{
${ }^{2}$ In general if $X$ is a right $G$ space and $Y$ is a left $G$ space $X \times{ }_{G} Y$ denotes the space obtained from $X \times Y$ by identifying $\left(x g, g^{-1} y\right)$ with $(x, y) . x \in X, y \in Y, g \in G$.
} 
and a commutative diagram

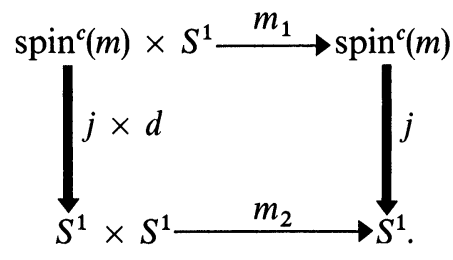

Here $m_{1}$ is multiplication in $\operatorname{spin}^{c}(m), m_{2}$ multiplication in $S^{1}$ and $d$ is the squaring map $d(t)=t^{2}$. Since $S^{1}$ is central in $\operatorname{spin}^{c}(m), m_{1}$ is a homomorphism of groups.

3. Spin ${ }^{c}$ bundles. Here we collect some of the properties of $\operatorname{spin}^{c}(m)$ bundles which will be useful in our analysis of actions on $\operatorname{spin}^{c}$ manifolds.

The classifying space of a group $G$ is denoted by $B_{G}$. From diagram (2.5) and the fact that $m_{1}$ and $m_{2}$ are homomorphisms of groups we obtain a commutative diagram

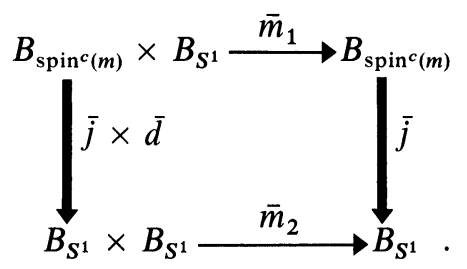

The map $\bar{m}_{1}$ makes $B_{\operatorname{spin}^{c}(m)}$ the total space of a principle $B_{S^{1}}$ bundle over $B_{S O(m)}$ and there is a commutative diagram of fiber spaces

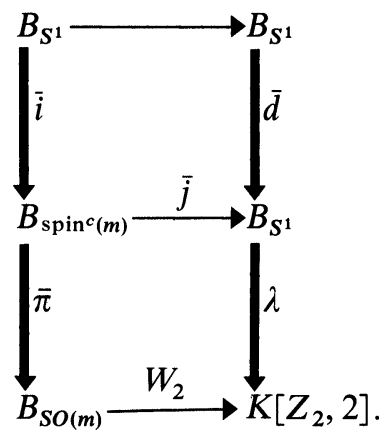

which shows that the principle bundle $\xi$ defined by $\bar{\pi}$ is induced from the bundle over $K\left[Z_{2}, 2\right]$ via the map $W_{2}$. Of course, the bundle over $K\left[Z_{2}, 2\right]$ arises from the diagram of groups $1 \rightarrow Z_{2} \rightarrow S^{1} \overleftrightarrow{\rightarrow} S^{1} \rightarrow 1$.

Principle $B_{S^{1}}$ bundles over $B_{S O(m)}$ induced from this principle $B_{S^{1}}$ bundle over $K\left[Z_{2}, 2\right]$ are classified by $H^{2}\left(B_{S O(m)}, Z_{2}\right) \cong Z_{2}$. This group is generated by the universal second Stiefel-Whitney class $W_{2}$. Thus, to justify the 
notation $W_{2}$ for the map inducing the bundle $\xi$, it suffices to show that $\pi_{2}\left(B_{\text {spin }^{c}(m)}\right)$ is not $Z \oplus Z_{2}=\pi_{2}\left(B_{S^{1}} \times B_{\text {SO }(m)}\right)$, i.e., that $W_{2}$ is not the trivial map. But this follows from the fact that $\pi_{1}\left(\operatorname{spin}^{c}(m)\right)=Z$ which is a consequence of the fact that $\operatorname{spin}(m)$ is simply connected and the exact sequence (2.4). This gives

LEMMA 3.3. $B_{\text {spin }^{c}(m)}$ is the total space of a principle $B_{S^{1}}$ bundle over $B_{S O(m)}$ induced from the nontrivial bundle over $K\left[Z_{2}, 2\right]$ by a map $W_{2}: B_{S O(m)}$ $\rightarrow K\left[Z_{2}, 2\right]$ realizing the universal second Stiefel-Whitney class.

Let $\delta$ be a principle $S O(m)$ bundle over a space $X$ classified by a.map $c: X \rightarrow B_{S O(m)}$. By definition a $\operatorname{spin}^{c}(m)$ structure (briefly a $\operatorname{spin}^{c}$ structure) on $\delta$ is a homotopy class of maps $\tilde{c}: X \rightarrow B_{\operatorname{spin}^{c}(m)}$ such that $\tilde{\pi} \tilde{c}$ is homotopic to $c$. Let $\sigma \in H^{2}\left(B_{S^{1}}, Z\right)$ be a generator of this group.

Lemma 3.4. The $\bmod 2$ reduction of $\tilde{c}^{*} j^{*}(\sigma)$ is $W_{2}(\delta)$, the second StiefelWhitney class of $\delta$.

Proof. Let $\sigma_{2}$ be the mod 2 reduction of $\sigma$. Then if $i \in H^{2}\left(K\left[Z_{2}, 2\right], Z_{2}\right)$ is the generator, $\lambda^{*}(i)=\sigma_{2}$ and

$$
W_{2}(\delta)=c^{*} W_{2}^{*}(i)=\tilde{c}^{*} \bar{\pi}^{*} W_{2}^{*}(i)=\tilde{c}^{*} \bar{j}^{*} \sigma_{2}
$$

which is the mod 2 reduction of $\left(\tilde{c}^{*} \tilde{j}^{*} \sigma\right)$.

We remark that the multiplication $\bar{m}_{2}$ of (3.1) corresponds to the tensor product of complex line bundles. Since $B_{\operatorname{spin}^{c}(m)}$ is the total space of a principle $B_{S^{1}}$ action, $H^{2}(X, Z)$ acts on $\left[X, B_{\operatorname{spin}^{c}(m)}\right]$, the set of homotopy classes of maps of $X$ to $B_{\text {spinc }}(m)$, in the following manner. Let $f \in\left[X, B_{\operatorname{spin}^{c}(m)}\right]$ and $g \in\left[X, B_{S^{1}}\right]=H^{2}(X, Z)$. Then we obtain a commutative diagram

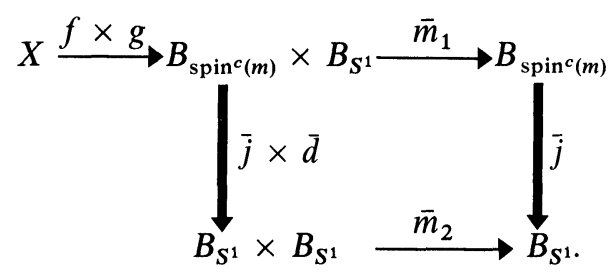

Denote the composition $\bar{m}_{1} f \times g$ by $f \circ g$. This defines the action of $H^{2}(X, Z)$ on $\left[X, B_{\operatorname{spin}^{c}(m)}\right]$.

Let $\hat{f}$ denote the complex line bundle over $X$ defined by $\bar{j} f$. If $P_{f}$ denotes the principle $\operatorname{spin}^{c}(m)$ bundle over $X$ induced by $f$, then $\hat{f}=P_{f} \times{ }_{\operatorname{spin}^{c}(m)} C$ where $\operatorname{spin}^{c}(m)$ acts on $C$ via the representation $j$ of $\operatorname{spin}^{c}(m)$ to $S^{1}$ given by (2.4). Let $\hat{g}$ denote the complex line bundle over $X$ determined by $g$.

Lemma 3.5. $(f \circ g)^{\wedge}=\hat{f} \cdot \hat{g}^{2}$. 
ProOF. $(f \circ g)^{\wedge}=\bar{j} \bar{m}_{1}(f \times g)=\bar{m}_{2}(\bar{j} \times \bar{d})(f \times g)=\bar{m}_{2}(\bar{j} f \times \bar{d} g)=\hat{f} \cdot \hat{g}^{2}$ Since $B_{\text {spinc }^{c}(m)}$ is the fiber product of

$$
B_{\mathrm{SO}(m)} \stackrel{w_{2}}{\longrightarrow} K\left[Z_{2}, 2\right] \text { and } B_{S^{1}} \stackrel{\lambda}{\longrightarrow} K\left[Z_{2}, 2\right]
$$

we have:

COROLlaRY 3.6. The $\operatorname{spin}^{c}(m)$ structures on a principle $S O(m)$ bundle $\delta$ are in 1-1 correspondence with elements $d \in H^{2}(X, Z)$ whose $\bmod 2$ reduction is $W_{2}(\delta)$ the second Stiefel-Whitney class of $\delta$. An explicit correspondence is this: Let $P$ be the total space of a principle $\operatorname{spin}^{c}(m)$ bundle such that the orbit space $P / S^{1}=Q$ of $P$ by $S^{1} \subset \operatorname{spin}^{c}(m)$ is the total space of $\delta$. (Since $\operatorname{spin}^{c}(m) / S^{1}=S O(m), Q$ is the total space of a principle $S O(m)$ bundle. $)$ Then the correspondence is given by $P \rightarrow c_{1}(\xi)$. Here $\xi$ is the line bundle whose total space is $P \times_{\operatorname{spin}^{c}(m)} C$ and $c_{1}(\xi)$ is its first Chern class.

Suppose that $X$ is a smooth $m$ dimensional manifold. By definition a $\operatorname{spin}^{c}(m)$ structure on $X$ is a $\operatorname{spin}^{c}(m)$ structure on its tangent bundle $T X$.

Lemma 3.7. If $H^{3}\left(X, Z_{2}\right)=0$ then $X$ has a $\operatorname{spin}^{c}(m)$ structure.

ProOF. $H^{2}(X, Z) \rightarrow H^{2}\left(X, Z_{2}\right)$ is onto.

REMARK. We find it convenient at times to use the total space $P$ of a principle $\operatorname{spin}^{c}(m)$ bundle to designate the $\operatorname{spin}^{c}(m)$ structure it defines.

4. Orientation of $G$ manifolds and Poincaré duality. Let $X$ be a compact $G$ manifold of dimension $m$. Let $W$ be a (real) $G$ vector bundle over $X$ of dimension $k$. An orientation for $W$ is a class $\omega_{G} \in K_{G}^{k}(W)$ such that $i^{*} \omega_{G} \in K_{G}^{k}(W \mid O)$ generates $K_{G}^{*}(W \mid O)$ freely over $K_{G}^{*}(O)$ for every orbit $O$. Here $i$ is the inclusion of $W \mid O$ in $W$.

Definition. An orientation for $X$ is an orientation $\alpha_{G} \in K_{G}^{m}(T X)$ of the tangent bundle of $X$.

Observe that if $X$ has a boundary $\partial X$, then $\partial X$ is oriented by $j^{*}\left(\alpha_{G}\right)$, $j: \partial X \rightarrow X$ because

$$
K_{G}^{m}\left(\left.T X\right|_{\partial X}\right)=K_{G}^{m}\left(T \partial X \times R^{1}\right)=K_{G}^{m-1}(T \partial X) .
$$

An orientation class $\alpha_{G}$ provides a Thom homomorphism $\psi=\psi_{G}^{X}: K_{G}^{*}(X)$ $\rightarrow K_{G}^{*}(T X), \psi_{G}^{X}(\lambda)=\alpha_{G} \cdot \lambda$.

LEMMA 4.1. $\psi_{G}^{X}$ is an isomorphism.

Proof. Let $\bar{X}$ denote the orbit space of $X$ by $G$. There are two sheaves over $\bar{X}, \mathscr{S}_{q}$ and $\mathscr{T}_{q}$ whose stalks are respectively

$$
\mathscr{S}_{q}(\bar{x})=K_{G}^{q}(G x), \quad \mathscr{T}_{q}(\bar{x})=K_{G}^{q}(T G x),
$$

where $\bar{x} \in \bar{X}$ and $G x \subset X$ is the orbit of $x \in X$ lying over $\bar{x}$. 
Multiplication by $\alpha_{G}$ induces a map of the spectral sequence [12]

$$
E_{2}^{p, q}=H^{p}\left(\bar{X}, \mathscr{S}_{q}\right) \Rightarrow K_{G}^{*}(X)
$$

to the spectral sequence

$$
E_{2}^{p, q}=H^{p}\left(\bar{X}, \mathscr{T}_{q}\right) \Rightarrow K_{G}^{*}(T X)
$$

which is an isomorphism on the $E_{2}$ level.

Corollary 4.2. $\psi_{G}^{\partial X}$ is an isomorphism.

CoROllary 4.3. $\psi_{G}^{(X, \partial X)}: K_{G}^{*}(X, \partial X) \rightarrow K_{G}^{*}\left(T X,\left.T X\right|_{\partial X}\right)$ is an isomorphism.

Proof. Multiplication by $\alpha_{G}$ induces a map of the exact sequence of the pair $(X, \partial X)$ to $\left(T X,\left.T X\right|_{\partial X}\right)$ which is an isomorphism on two terms by the preceding. The result follows by the five lemma.

REMARK. We could equally well have defined an orientation for $X$ by means of a class $\beta_{G} \in K_{G}^{*}(N X)$ where $N X$ is the normal bundle of $X$ which is equivariantly imbedded in a complex representation space $M$ for $G$. These are equivalent concepts.

The significance of this remark is that $K_{G}^{*}(N X)$ is the equivariant homology of $X$ dual to $K_{G}^{*}(X)$ if $X$ is a closed manifold. To see this, note that $X \subset M \subset M^{+}=S^{2 n}$ where $M^{+}$is the one point compactification of $M$ which we assume has complex dimension $n$. Then by definition

$$
K_{i}^{G}(X)=K_{G}^{2 n-i}\left(S^{2 n}, S^{2 n}-X\right) \cong K_{G}^{2 n-i}\left(N X,\left.N X\right|_{\partial X}\right)
$$

by excision.

The map $C$ which collapses the exterior of the closure $\overline{N X}$ of $N X$ in $M^{+}$ induces

$$
C^{*}: K_{G}^{*}(\overline{N X}, \partial \overline{N X}) \rightarrow K_{G}^{*}(M,+)=R(G) .
$$

The composition of $C^{*}$ with the map $m: K_{G}^{*}(X) \otimes K_{G}^{*}\left(N X,\left.N X\right|_{\partial X}\right)$ $\rightarrow K_{G}^{*}\left(N X,\left.N X\right|_{\partial X}\right)$ which exhibits $K_{G}^{*}\left(N X,\left.N X\right|_{\partial X}\right)$ as a module over $K_{G}^{*}(X)$ defines the duality pairing

$$
\hat{d}: K_{G}^{i}(X) \otimes K_{m-i}^{G}(X) \rightarrow R(G) .
$$

There is a second duality pairing which is more appropriately related to our purpose when $X$ is a closed manifold. It is the map

$$
\hat{d}: K_{G}^{*}(X) \otimes K_{G}^{*}(T X) \rightarrow R(G)
$$

which is defined by

$$
\hat{d}(x \otimes y)=\operatorname{Id}_{G}^{X}(x \cdot y)
$$

for $x \in K_{G}^{*}(X)$ and $y \in K_{G}^{*}(T X)$. 
REMARK. When $X$ is a closed manifold, these two pairings are the same. The point is that the pairing $d$ is more generally defined while the pairing $\hat{d}$ is more accessible to computation because of the properties of the index homomorphism. To extend the definition of $d$ to spaces $X$ which are not manifolds we must assume that $X$ is imbedded in a complex representation space $M$ of $G$ with an equivariant regular neighborhood $\overline{N X} \subset M^{+}$. Then $K_{i}^{G}(X)=K_{G}^{2 n-i}(\overline{N X}, \partial \overline{N X})$ and $d$ is defined for such $G$ spaces $X$ as above.

To justify the above remark we offer

Proposition 4.5. If $X$ is a closed $G$ manifold there is an isomorphism $\phi: K_{G}^{*}(T X) \rightarrow K_{G}^{*}(N X)$ which takes $\hat{d}$ to $d$.

Proof. There is a commutative diagram of vector bundles

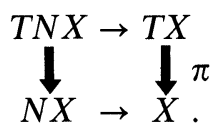

Since $N X \subset M$ is an open subset, $T N X \cong N X \times M$ as a $G$ vector bundle over $N X$. However, $T N X \cong \pi^{*}(N X \otimes C)$. Since $T N X$ is a complex $G$ bundle over $N X$ as well as over $T X$, we have Thom isomorphisms

$$
\begin{aligned}
& \psi_{1}: K_{G}^{*}(N X) \rightarrow K_{G}^{*}(T N X), \\
& \psi_{2}: K_{G}^{*}(T X) \rightarrow K_{G}^{*}(T N X),
\end{aligned}
$$

and a commutative diagram

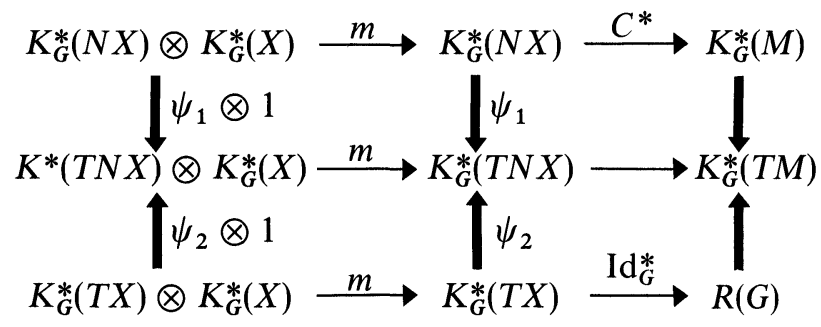

in which all vertical maps are isomorphisms. Since $d$ is defined by the top row and $\hat{d}$ by the bottom, the demonstration is complete.

With the equivariant homology of a manifold $X$ defined by (4.4) we obtain Poincare duality free if an orientation is given.

Proposition 4.6. If $X$ is a compact oriented $G$ manifold of dimension $m$, then $X$ satisfies Poincaré duality: $K_{i}^{G}(X) \cong K_{G}^{m-i}(X, \partial X)$.

Proof. If $\alpha_{G} \in K_{G}^{m}(T X)$ is an orientation then

$$
\psi^{X}: K_{G}^{*}(X, \partial X) \rightarrow K_{G}^{*}\left(T X,\left.T X\right|_{\partial X}\right)
$$


is an isomorphism. But

$$
K_{G}^{*}\left(T X,\left.T X\right|_{\partial X}\right) \cong K_{G}^{*}\left(N X,\left.N X\right|_{\partial X}\right)=K_{*}^{G}(X) .
$$

In view of Proposition 4.6, we expect the difficulties of studying $G$ actions on manifolds by using Poincaré duality to be intimately connected with the existence of an orientation. There is a very general situation in which it is often possible to construct an orientation. This occurs when $X$ is a $\operatorname{spin}^{c}(m)$ manifold. Again this means there is a principle $\operatorname{spin}^{c}(m)$ bundle $P$ over $X$ such that

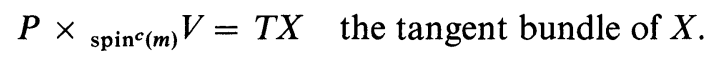

We assume: that $G$ acts on the left on $P$, commutes with the right action of $\operatorname{spin}^{c}(m)$ and is compatible with the natural left action on $Q$, the principle $S O(m)$ bundle associated to $T X$, (in other words, the frame bundle of $T X$ ), obtained by sending $\left(g,\left[v_{1}, v_{2}, \ldots, v_{m}\right]\right) \rightarrow\left[d g v_{1}, d g v_{2}, \ldots, d g v_{m}\right]$ for $g \in G,\left[v_{1}, \ldots, v_{m}\right]$ a frame in $Q$ and $d g$ the differential of $G$.

The elliptic pairing (2.2) is the basic property for constructing a class $\delta_{G}$ in $K_{G}^{m}(T X)$ from the equivariant $\operatorname{spin}^{c}(m)$ structure on $X$.

For brevity, set $H=\operatorname{spin}^{c}(m)$. We define a $G \times H$ complex of vector bundles [6, p. 489] over $P \times V$ :

$$
\begin{gathered}
P \times V \times \Delta_{+} \stackrel{\Phi}{\longrightarrow} P \times V \times \Delta_{-}, \\
\Phi(p, v, \delta)=(p, \theta(v, \delta)) .
\end{gathered}
$$

The $G \times H$ action on $P \times V$ is given by

$$
(g, h)(p, v)=\left(g p h^{-1}, h v\right)
$$

the action on $P \times V \times \Delta_{ \pm}$is given by

$$
(g, h)(p, v, b)=\left(g p h^{-1}, h v, h b\right) .
$$

Since $\theta$ is an elliptic pairing, this complex defines an element

$$
\delta_{G} \in K_{G \times H}^{*}(P \times V)=K_{G}^{m}\left(P \times_{H} V\right)=K_{G}^{m}(T X) .
$$

Here are some examples in which $\delta_{G}$ or a close variant defines an orientation.

EXAMPLE 1. $X$ is a point, $m$ is even, $G=U(m / 2)$ is the unitary group of isometries of $C^{m / 2}$ and $\hat{V}=C^{m / 2}$ denotes the standard $U(m / 2)$ module. It is a $U(m / 2)$ bundle over a point. We define an orientation class $\Delta_{U}$ for $\hat{V}$.

The elliptic pairing $\theta: V \times \Delta_{+} \rightarrow V \times \Delta_{-}$of (2.2) gives an elliptic $U(m / 2)$ complex

$$
\hat{\theta}: \hat{V} \times \Delta_{+} \rightarrow \hat{V} \times \Delta_{-}
$$


over a point. Here $\Delta_{+}$and $\Delta_{-}$are $U(\mathrm{~m} / 2)$ modules via the homomorphism $\psi_{0}$ of (2.3). This complex defines an element $\Delta_{U} \in K_{U}^{*}(\hat{V})$.

Proposition 4.8. $K_{U}^{*}(\hat{V})$ is a free module over $R(U)=K_{U}^{*}(\mathrm{pt})$ generated by $\Delta_{U}$.

Proof. The symbol of the de Rham complex of $\hat{V}, \lambda_{\hat{V}} \in K_{U}^{*}(\hat{V})$ is a generator [6], so

$$
\Delta_{U}=a \cdot \lambda_{\hat{V}} \in K_{U}^{*}(\hat{V})
$$

for some $a \in R(U)$. Let $j: \mathfrak{T} \rightarrow U$ denote the inclusion of the maximal torus and $\hat{j}$ the composition

$$
K_{U}^{*}(\hat{V}) \stackrel{i^{*}}{\rightarrow} K_{U}^{*}(0) \stackrel{j^{*}}{\rightarrow} K_{T}^{*}(0)=R(\mathfrak{I}) .
$$

Here $i^{*}$ is the restriction defined by the inclusion of the origin 0 . Now

$$
\begin{gathered}
\hat{j} \lambda_{\hat{V}}=\lambda_{-1}(\hat{V})=\prod_{i=1}^{m / 2}\left(1-t_{i}\right) \in R(\mathfrak{I}), \\
R(\mathfrak{I})=Z\left[t_{1}, t_{1}^{-1}, t_{2}, t_{2}^{-1}, \ldots, t_{m / 2}, t_{m / 2}^{-1}\right] .
\end{gathered}
$$

But $\hat{j} \Delta_{U}=\left.\left(\Delta_{+}-\Delta_{-}\right)\right|_{T}=\prod\left(1-t_{i}^{-1}\right) t_{i}$. This follows from the definition of $\psi_{0}$ and the fact that the trace of $t=\left(t_{1}, t_{2}, \ldots, t_{m / 2}\right)$ acting on $\Delta_{+}$minus the trace of $t$ on $\Delta_{-}$is $\prod\left(1-t_{i}^{-1}\right) t_{i}$. Since $R(U) \rightarrow R(\mathfrak{I})$ is injective, $a=(-1)^{m / 2}$.

If $G \stackrel{\rho}{\rightarrow} U(m / 2)$ is a representation then $\Delta_{G} \in K_{G}^{*}(\hat{V})$, is defined to be $\rho^{*} \Delta_{U}$. It generates $K_{G}^{*}(\hat{V})$ freely over $R(G)$.

EXAMPLE 2. $X$ is a simply connected $\operatorname{spin}^{c}(m)$ manifold with an $S^{1}$ action which satisfies (4.7).

Proposition 4.9. $\delta_{S^{1}}$ is an orientation for $X$.

Proof. There are two kinds of orbits, a point and $S^{\mathbf{1}}$. If $p$ is a fixed point and $i:\left.T X\right|_{p} \rightarrow T X$ the inclusion, $\left.T X\right|_{p}=\hat{V}$ as an $S^{1}$ bundle over $p$ and

$$
i^{*} \delta_{S^{1}}=\Delta_{S^{1}} \in K_{S^{1}}^{*}(\hat{V}) \text {. }
$$

If $S^{1}$ is an orbit, then because $X$ is simply connected

$$
\left.P\right|_{S^{1}} \cong S^{1} \times \operatorname{spin}^{c}(m)
$$

is the restriction of the principle $\operatorname{spin}^{c}(m)$ bundle over $X$ to the orbit $S^{1}$. If $x$ denotes the point $S^{1} / S^{1}$, then $K_{S^{1}}^{*}\left(S^{1}\right)=K^{*}(x)$ and

$$
K_{S^{1}}^{*}\left(\left.T X\right|_{S^{1}}\right)=K_{S^{1}}^{*}\left(S^{1} \times \hat{V}\right) \cong K^{*}(\hat{V})
$$

and $i^{*}\left(\delta_{S^{1}}\right)=\Delta_{1} \in K^{*}(\hat{V})$ is an orientation for $\hat{V}$ over $x$. Here 1 denotes the trivial group. 
EXAMPLE 3. $X=U(Z)$ is the group of isometries of a complex $G$ module $Z$ of real dimension $m$. So as a space $X=U(m / 2)$. The $G$ module structure on $Z$ gives a representation $\rho$ of $G$ in $U(Z)$ and $G$ acts on $X=U(Z)$ via inner automorphisms. Then

$$
T U(Z)=U(Z) \times M
$$

where $M$ is the real $G$ module $\rho^{*} M^{1}$ and $M^{1}$ is the tangent space of $U(Z)$ at the identity. It is a real $U(Z)$ module via the adjoint representation of $U(Z)$ on $M^{1}$. We emphasize the fact that $(4.10)$ is the expression of $T U(Z)$ as a $G$ vector bundle over $U(Z)$.

Either $M^{1}$ or $M^{1} \times R^{1}$, depending on the dimension of $M^{1}$, is a complex $U(Z)$ module $\hat{V}$. Then restricting to $G$ we obtain a complex of $G$ vector bundles

$$
U(Z) \times \hat{V} \times \Delta_{+} \stackrel{1 \times \hat{\theta}}{\longrightarrow} U(Z) \times \hat{V} \times \Delta_{-}
$$

whose symbol $\alpha_{G} \in K_{G}^{0}(U(Z) \times \hat{V})$ is

$$
1 \otimes \Delta_{G} \in K_{G}^{*}(U(Z)) \otimes_{R(G)} K_{G}^{*}(\hat{V})=K_{G}^{*}(U(Z) \times \hat{V})
$$

by the Künneth theorem of $[\mathbf{1 0}]$. Since $U(Z) \times \hat{V}$ is either $T U(Z)$ or $T U(Z) \times R^{1}$ as a $G$ space, $\alpha_{G} \in K_{G}^{*}(T U(Z))$ and

Proposition 4.11. $\alpha_{G}$ is an orientation.

Proof. This follows from the fact that $\alpha_{G}=1 \otimes \Delta_{G}$ and Proposition 4.8 together with the Künneth theorem.

EXAMPLE 4. $X=G / H$ where $H$ and $G$ are compact connected Lie groups, $H$ has maximal rank in $G$ and $G / H$ has a spinc structure. $G$ acts on $G / H$ by left translation. Then the $G$ action on $X$ satisfies the condition of (4.7) and the class $\alpha_{G}$ constructed from the $\operatorname{spin}^{c}$ structure is an orientation for $X$. We omit details of the proof.

5. The homomorphism $K_{G}^{*}(X) \stackrel{\psi_{G}}{\longrightarrow} K_{G}^{*}(T X) \stackrel{\operatorname{Id}_{G}^{X}}{\longrightarrow} R(G)$. When $X$ is oriented we can consider the composition $\operatorname{Id}_{G}^{X} \psi_{G}=\tau_{G}^{X}$. It provides powerful invariants for the $G$ action on $X$. The most important case for our purpose occurs when $X$ has a $\operatorname{spin}^{c}(m)$ structure ( $m$ even) and is oriented by $\delta_{G}$. We require an explicit formula for $\operatorname{Id}_{G}^{X}\left(\delta_{G}\right)$ in terms of the representations of $G$ in the normal fibers to the fixed point sets.

We make these assumptions:

(5.0) $G$ is topologically cyclic, i.e., has a dense generator $g$ and for each component $X_{i}^{G}$ of the fixed point set of $G, X^{G}$, we have $H^{3}\left(X_{i}^{G}, Z_{2}\right)=0$. In addition we assume that the action of $G$ on $X$ satisfies (4.7). 
We now investigate the behavior of the class $\delta_{G}$ when restricted to $K_{G}^{*}\left(T X^{G}\right)$. Over $X_{j}^{G}$ we have a diagram of bundles

$$
\begin{aligned}
& \left.P_{0} \subset P\right|_{X_{j}^{G}}=\bar{P} \\
& \downarrow \\
& \left.Q_{0} \subset Q\right|_{X_{j}^{G}}=\bar{Q}
\end{aligned}
$$

where $\bar{Q}$ is the principle $S O(m)$ bundle associated to $\left.T X\right|_{X^{G}}$ and $Q_{0}$ is the principle $S O\left(k_{j}\right) \times S O\left(m-k^{j}\right)$ associated to $T X_{j}^{G} \oplus N X_{j}^{G^{j}} \cdot Q_{0}$ is a reduction of $\bar{Q}$. Here we assume $X_{j}^{G}$ is orientable so its dimension $k_{j}$ is even as is $m$. The group of the bundle $P_{0}$ is $\operatorname{spin}^{c}\left(k_{j}\right) \times_{S^{1}} \operatorname{spin}^{c}\left(m-k_{j}\right)=H_{0}$. For convenience of exposition, we concentrate on a particular component of $X^{G}$ and drop the subscript $j$. We are interested in the class $T i^{*} \delta_{G} \in K_{G}\left(T X^{G}\right)$; $T i: T X^{G} \rightarrow T X$ is the inclusion. Note that $T i$ is the composition

$$
T X^{G} \stackrel{S}{\rightarrow} T X^{G} \oplus N X^{G} \stackrel{j}{\rightarrow} T X
$$

where $S$ is the zero section of $T X^{G} \oplus N X^{G}$ as a bundle over $T X^{G}$ and $j$ is the inclusion.

Let $d \in H^{2}(X)$ be the class associated to the principle $\operatorname{spin}^{c}(m)$ bundle $P$ over $X$. See Corollary 3.6. Since $H^{3}\left(X^{G}, Z_{2}\right)=0$, every element of $H^{2}\left(X^{G}, Z_{2}\right)$ is the reduction of an element of $H^{2}\left(X^{G}, Z\right)$. Choose $d_{1} \in H^{2}\left(X^{G}, Z\right)$ whose reduction is $W_{2}\left(X^{G}\right)$ and let $d_{2}=f^{*} d-d_{1}$; $f: X^{G} \rightarrow X$ the inclusion. The principle $\operatorname{spin}^{c}(k)$ and $\operatorname{spin}^{c}(m-k)$ bundles determined by $d_{1}$ and $d_{2}$ are denoted by $P_{1}$ and $P_{2}$. Then $P_{1} \times P_{2}$ is the total space of a principle $\hat{H}_{0}=\operatorname{spin}^{c}(k) \times \operatorname{spin}^{c}(m-k)$ bundle over $X \times X$ whose orbit space $P_{1} \times{ }_{S^{1}} P_{2}$ by the diagonal action $g(x, y)$ $=\left(g^{-1} x, g y\right), g \in S^{1}, x \in P_{1}, y \in P_{2}$, is the total space of a principle $H_{0}$ bundle over $X^{G} \times X^{G}$. If $D: X^{G} \rightarrow X^{G} \times X^{G}$ denotes the diagonal map then $D^{*} P_{1} \times{ }_{S^{1}} P_{2}=P_{0}$.

Now we remark that

$$
\bar{P} \times_{\operatorname{spin}^{c}(m)} V \times \Delta_{ \pm}=P_{0} \times_{H_{0}} V \times \Delta_{ \pm} .
$$

The homomorphism $\operatorname{spin}^{c}(k) \times \operatorname{spin}^{c}(m-k) \rightarrow \operatorname{spin}^{c}(m)$ makes $\Delta_{ \pm}$and $V \hat{H}_{0}$ modules and these decompose as

$$
\begin{gathered}
V=V^{1} \oplus V^{2}, \\
\Delta_{+}=\Delta_{+}^{1} \cdot \Delta_{+}^{2} \oplus \Delta_{-}^{1} \cdot \Delta_{-}^{2}, \quad \Delta_{-}=\Delta_{+}^{1} \cdot \Delta_{-}^{2} \oplus \Delta_{-}^{1} \cdot \Delta_{+}^{2},
\end{gathered}
$$

where $\cdot$ denotes tensor product. Here the superscript 1 denotes a $\operatorname{spin}^{c}(k)$ module and a superscript 2 a $\operatorname{spin}^{c}(m-k)$ module.

Next we observe that

$$
P_{0} \times_{H_{0}} V \times \Delta_{ \pm}=\hat{P}_{0} \times_{\hat{H}_{0}} V \times \Delta_{ \pm}
$$


where $\hat{P}_{0}=D^{*} P_{1} \times P_{2}$. From these observations we have

$$
j^{*} \delta_{G}=\widetilde{D}^{*} \gamma_{G} \hat{\otimes} \theta_{G}=\gamma_{G} \cdot \theta_{G} \in K_{G}^{*}\left(T X^{G} \oplus N X^{G}\right) .
$$

Here $\gamma_{G} \hat{\otimes} \theta_{G} \in K_{G}^{*}\left(T X^{G} \times N X^{G}\right)$ is the external tensor product of the class $\gamma_{G} \in K_{G}^{*}\left(T X^{G}\right)$ defined by the complex

$$
P_{1} \times_{\operatorname{spin}^{c}(k)} V^{1} \times \Delta_{+}^{1} \rightarrow P_{1} \times_{\operatorname{spin}^{c}(k)} V^{1} \times \Delta_{-}^{1},
$$

$\theta_{G} \in K_{G}^{*}\left(N X^{G}\right)$ is the class defined by the complex

$$
P_{2} \times_{\operatorname{spin}^{c}(m-k)} V^{2} \times \Delta_{+}^{2} \rightarrow P_{2} \times_{\operatorname{spin}^{c}(m-k)} V^{2} \times \Delta_{-}^{2}
$$

and $\widetilde{D}: T X^{G} \oplus N X^{G} \rightarrow T X^{G} \times N X^{G}$ is the bundle map covering the diagonal map $D$. Now $P_{2} \times{ }_{\operatorname{spin}^{c}(m-k)} V^{2} \times \Delta_{ \pm}^{2} \times \omega_{ \pm}$are vector bundles over $X^{G}$ and $S^{*} \gamma_{G} \theta_{G}=\gamma_{G} \cdot\left(\omega_{+}-\omega_{-}\right)$hence

$$
T i^{*} \delta_{G}=S^{*} j^{*} \alpha_{G}=\gamma_{G}\left(\omega_{+}-\omega_{-}\right) \in K_{G}^{*}\left(T X^{G}\right) .
$$

We record this as

Lemma 5.1. Let $G$ be cyclic and $H^{3}\left(X^{G}, Z_{2}\right)=0$. Let $d \in H^{2}(X, Z)$ determine a $\operatorname{spin}^{c}(m)$ structure $P$ on $X$. Choose $d_{1} \in H^{2}\left(X^{G}, Z\right)$ whose $\bmod 2$ reduction is $W_{2}\left(X^{G}\right)$ and let $d_{2}=f^{*}(d)-d_{1}, f: X^{G} \rightarrow X$ the inclusion. Then $d_{1}$ and $d_{2}$ determine spin ${ }^{c}$ structures $P_{1}$ and $P_{2}$ on $T X^{G}$ and $N X^{G}$ and we obtain $G$ vector bundles $\omega_{+}, \omega_{-} \in K_{G}^{*}(X)$ coming from $P_{2}$ and a class $\gamma_{G} \in K_{G}^{*}\left(T X^{G}\right)$ coming from $P_{1}$ such that

$$
T i^{*} \delta_{G}=\gamma_{G}\left(\omega_{+}-\omega_{-}\right) \in K_{G}^{*}\left(T X^{G}\right)
$$

where $\delta_{G}$ is the orientation class associated to the principle $\operatorname{spin}^{c}(m)$ bundle $P$.

Now the action of $G$ on $N X^{G}$ gives a representation $\rho: G \rightarrow S O(m-k)$. Namely if $x \in X^{G}$ then $g x=x$; so, for $q \in Q_{0}$,

$$
\rho(g)=\operatorname{diag}\left(\begin{array}{cc}
g q=q \rho(g), \\
\cos \theta_{i} & \sin \theta_{i} \\
-\sin \theta_{i} & \cos \theta_{i}
\end{array}\right) \in S O(m-k),
$$

$i=1,2, \ldots,(m-k) / 2$. The numbers $\theta_{i}$ are well defined modulo $\pi=3.1416 \ldots$.

The action of $G$ on $P_{2}$ gives a homomorphism $\tilde{\rho}: G \rightarrow \operatorname{spin}^{c}(m-k)$ and a commutative diagram

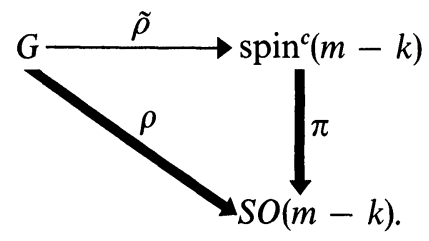


Moreover,

$$
\tilde{\rho}(g)=\left[\prod_{j=1}^{(m-k) / 2}\left(\cos \theta_{j} / 2-\sin \theta_{j} / 2 e_{2 j-1} e_{2 j}\right), \exp \left[-i\left(\sum \theta_{j} / 2+\lambda\right)\right]\right]
$$

for some $\lambda=\lambda(g), g \rightarrow e^{-i \lambda}$ is a homomorphism from $G$ to $S^{1}$ and $g p=p \tilde{\rho}(g)$ if $p$ lies over $x$; thus the action of $g$ on the fibers $\Delta_{+}^{2}$ and $\Delta_{-}^{2}$ of $\omega_{+}$and $\omega_{-}$is given by the representation $\tilde{\rho}$.

REMARK. Observe that if $X^{G}$ and hence $N X^{G}$ has $n$ components, we obtain $n$ representations $\tilde{\rho}_{i}, i=1,2, \ldots, n$, which completely describe the action of $G$ on the fibers of $\omega_{+}$and $\omega_{-}$.

The normal bundle $N X^{G}$ has a decomposition invariant under $G$,

$$
N X^{G}=N X^{G}(-1)+\sum_{u} N X^{G}(u),
$$

where the $u$ are complex numbers of absolute value 1 with positive imaginary part and $N X^{G}(u)$ has a complex structure in which $g \in G$ operates by multiplication by $u$. This gives a lifting $\hat{\rho}$ of the representation $\rho: G \rightarrow S O(m-k)$ to $U((m-k) / 2)$ and

$$
\hat{\rho}(g)=\operatorname{diag}\left\{v I_{v}\right\} \subset U((m-k) / 2) .
$$

$I_{v}$ is the $n_{v} \times n_{v}$ identity matrix where $n_{v}=\operatorname{dim}_{C} N(v), v=e^{i \theta}, 0 \leqq \theta<\pi$.

For a complex vector bundle $L$ of dimension $s$ and complex number $z$, set

$$
\begin{aligned}
\mathscr{F}(L, z) & =z^{-s / 2} \prod_{j=1}^{s}\left[z^{-1 / 2} e^{-y_{j} / 2}-z^{1 / 2} e^{y_{j} / 2}\right]^{-1} \\
& =\prod_{j=1}^{s}\left[e^{-y_{j} / 2}-z e^{y_{j} / 2}\right]^{-1}
\end{aligned}
$$

where the $y_{j}$ are the formal roots of the total Chern class of $L$. Observe that

$$
\mathscr{F}(L,-1)=\prod\left[e^{-y_{j} / 2}+e^{y_{j} / 2}\right]^{-1}
$$

also makes sense because this is a symmetric function of the $y_{j}^{2}$ and so is a function of the Pontrjagin classes of $N(-1)$.

Another cohomology class is defined by

$$
\hat{\mathscr{A}}\left(X^{G}\right)=\prod_{j} x_{j}\left(e^{x_{j} / 2}-e^{-x_{j} / 2}\right)^{-1} \in H^{*}\left(X^{G}, \boldsymbol{C}\right) .
$$

The elementary symmetric functions of the variables $x_{j}^{2}$ are the Pontrjagin classes of $X^{G}$.

We are now in position to give an explicit formula for $\operatorname{Id}_{G}^{X}\left[\delta_{G}\right](g) \in C$, i.e., the value of the character $\operatorname{Id}_{G}^{X}\left[\delta_{G}\right]$ at $g \in G$. 


\section{Proposition 5.2}

$$
\operatorname{Id}_{G}^{X}\left[\delta_{G}\right](g)=\sum_{j}\left(e^{f_{j}^{*}(d) / 2} \hat{A}\left(X_{j}^{G}\right) \prod_{u_{j}} \mathscr{F}\left(N X_{j}^{G}\left(u_{j}\right), u_{j}\right) e^{-i \lambda_{j}}\right)\left[X_{j}^{G}\right]
$$

The sum is over the components $X_{j}^{G}$ of $X^{G}$. The numbers $u_{j}$ are determined by the representation $\rho_{j}: G \rightarrow S O\left(m-k_{j}\right)$. Explicitly if

$$
\rho_{j}(g)=\operatorname{diag}\left\{\left(\begin{array}{rr}
\cos \theta_{l} & \sin \theta_{l} \\
-\sin \theta_{l} & \cos \theta_{l}
\end{array}\right)\right\} \subset S O\left(m-k_{j}\right),
$$

then the $u_{j}$ 's are the complex numbers $\left\{e^{i \theta_{l}}\right\}$ and $\operatorname{dim}_{C}\left(N X^{G}(u)\right)=$ number of $l$ with $e^{i \theta_{l}}=u$. The number $\lambda_{j}=\lambda_{j}(g)$ associated to the jth component is determined by the lifting $\tilde{\rho}_{j}: G \rightarrow \operatorname{spin}^{c}(m-k)$ via

$$
\tilde{\rho}_{j}(g)=\left[\prod_{l=1}^{(m-k) / 2} \cos \theta_{l} / 2-\sin \theta_{l} / 2 e_{2 l-1} e_{2 l}, \exp \left[-i\left(\sum \theta_{l} / 2+\lambda_{j}\right)\right]\right]
$$

and $g \rightarrow e^{i \lambda_{j}(g)}$ defines a homomorphism of $G$ to $S^{1}$. The inclusion of $X_{j}^{G}$ in $X$ is denoted by $f_{j}$ and the cohomology class in the sum is evaluated on the orientation class $\left[X_{j}^{G}\right]$. The class $d \in H^{2}(X, Z)$ determines the $\operatorname{spin}^{c}$ structure on $X$ used to define $\delta_{G}$.

Proof. $K_{G}^{*}\left(X^{G}\right)=R(G) \otimes K^{*}\left(X^{G}\right)$.

Let $c h_{g}: K_{G}^{*}\left(X^{G}\right) \rightarrow K^{*}\left(X^{G}, C\right)$ be defined by $\operatorname{ch}_{g} \chi \otimes u=\chi(g) \cdot \operatorname{ch}(u)$ for $\chi \in R(G)$ and $u \in H^{*}\left(X^{G}\right)$. Let $e \otimes \operatorname{Id}_{1}^{X^{G}} R(G) \otimes K^{*}\left(X^{G}\right) \rightarrow C \otimes \otimes_{Z} Z=C$ be defined by $e \otimes \operatorname{Id}_{1}^{X^{G}}(\chi \otimes u)=\chi(g) \cdot \operatorname{Id}_{1}^{X^{G}}(u)$. Let $T i: T X^{G} \rightarrow T X$ be the inclusion. Then

$$
\begin{aligned}
\operatorname{Id}_{G}^{X} \delta_{G}(g) & =e \otimes \operatorname{Id}_{1}^{X^{G}}\left(T i^{*} \delta_{G} / \lambda_{-1}\left(N X^{G} \otimes C\right)\right) \text { by [5] or Part I, (1.6), } \\
& =e \otimes \operatorname{Id}_{1}^{X^{G}}\left(\gamma_{G}\left\{\frac{\omega_{+}\left|X_{j}^{G}-\omega_{-}\right| X_{j}^{G}}{\lambda_{-1}\left(N X^{G} \otimes C\right)}\right\}\right), \quad \text { by Lemma 5.1, } \\
& =\sum_{j}\left[\frac{\left.c h_{1} \gamma_{G}\right|_{X_{j}^{G}} \mathscr{I}\left(X_{j}^{G}\right)}{\prod w_{j l}}\right] c h_{g}\left(\frac{\omega_{+}\left|X_{j}^{G}-\omega_{-}\right| X_{j}^{G}}{\lambda_{-1}\left(N X_{j}^{G} \otimes C\right)}\right)\left[X_{j}^{G}\right],
\end{aligned}
$$

where the $w_{j l}, l=1,2, \ldots$, are the formal roots of the Pontrjagin classes of $X_{j}$. This formula follows from [6, p. 559, Proposition 2.17]. Let $d_{1 j}$ and $d_{2 j}$ be the $j$ th components in $H^{2}\left(X^{G}, Z\right)=\prod_{j} H^{2}\left(X_{j}^{G}, Z\right)$ of the classes $d_{1}$ and $d_{2}$ discussed in Lemma 5.1. Then

$$
\begin{aligned}
\left.c h_{1} \gamma_{G}\right|_{X_{j}^{G}} & =e^{d_{1 j} / 2} \prod_{l} e^{w_{j l} / 2}-e^{-w_{j l} / 2}, \\
\mathscr{I}\left(X_{j}^{G}\right) & =\prod \frac{w_{j l}}{1-e^{w_{j l}}} \cdot \frac{-w_{j l}}{1-e^{-w_{j l}}}
\end{aligned}
$$


and

$$
\operatorname{ch}_{g}\left(\frac{\omega_{+}\left|X_{j}^{G}-\omega_{-}\right| X_{j}^{G}}{\lambda_{-1}\left(N X_{j}^{G} \otimes C\right)}\right)=e^{d_{2 j} / 2} e^{-i \lambda_{j}} \prod_{u_{j}} \mathscr{F}\left(N X^{G}\left(u_{j}\right), u_{j}\right) .
$$

Since $d_{1 j}+d_{2 j}=f_{j}^{*}(d)$, the result follows.

We can give a better geometric interpretation of the numbers $\lambda_{j}$ as follows. Let

$$
\omega=P \times{ }_{\operatorname{spin}^{c}(m)} C
$$

be the line bundle over $X$ determined by the representation $\operatorname{spin}^{c}(m) \rightarrow S^{1}$ which sends $[g, t]$ to $t^{2}$. Then $\omega$ is a $G$ bundle over $X$. Here $G$ acts on $\omega$ via

$$
g[p, c]=[g p, c], \quad p \in P, c \in C .
$$

The restriction of $\omega$ to the component $X_{j}^{G}$ defines a one dimensional complex representation of $G$, namely the representation of $G$ in the fibers of $\omega$. So if $x \in X_{j}^{G}$ and $\omega_{x}$ is the fiber over $x$, then $g$ operates on $\omega_{x}$ by multiplication by $e^{i \omega_{j}}$.

Proposition 5.3: The numbers $\omega_{j}$ defined by the restriction of the line bundle $\omega$ to the component $X_{j}^{G}$ by

$$
\left.\omega\right|_{p_{j}}(g)=e^{i \omega_{j}}, \quad p_{j} \in X_{j}^{G},
$$

and the numbers $\lambda_{j}$ and $\theta_{j l}$ defined by the representation of $G$ on $N X_{j}^{G}$ by

$$
\begin{gathered}
\tilde{\rho}_{j}: G \rightarrow \operatorname{spin}^{c}\left(m-k_{j}\right), \\
\tilde{\rho}_{j}(g)=\left[\prod_{l}\left(\cos \theta_{j l} / 2-\sin \theta_{j l} / 2 e_{2 l-1} e_{2 l}, \exp \left[-i\left(\sum_{l} \theta_{j l} / 2+\lambda_{j}\right)\right]\right],\right.
\end{gathered}
$$

are related by

$$
-\left(\sum_{l} \theta_{j l}+2 \lambda_{j}\right)=\omega_{j}
$$

Proof. If $x \in X_{j}^{G}$, then $g x=x$; so if $p \in P$ lies over $x$, then

$$
g p=p \tilde{\rho}_{j}(g)
$$

and

$$
\begin{aligned}
g[p, c] & =\left[p \rho_{j}(g), c\right]=\left[p, \rho_{j}(g) c\right] \\
& =\left[p, \exp \left[-i\left(\sum \theta_{j l}+2 \lambda_{j}\right)\right] c\right]
\end{aligned}
$$

and this shows that the representation of $G$ on $\omega_{x}$ has character value, at $g \in G, \exp \left[-i\left(\sum \theta_{j l}+2 \lambda_{j}\right)\right]$. 
REMARK. More generally for $u \in K_{G}^{*}(X)$,

$$
\operatorname{Id}_{G}^{X}\left(\delta_{G} \cdot u\right)(g)=\sum_{j} c h_{g}\left(u \mid X_{j}^{G}\right) \cdot A_{j}\left[X_{j}^{G}\right], \quad g \in G
$$

where $A_{j}$ is the expression in parentheses occurring in Proposition 5.2. In particular when $g=1 \in G$,

$$
\operatorname{Id}_{G}^{X}\left(\delta_{G} \cdot u\right)(1)=\operatorname{ch}(u) e^{d / 2} \hat{\mathscr{A}}(X)[X] .
$$

An important special case occurs when $G=S^{1}$ and the fixed point set $X^{G}$ consists of isolated points $p_{j}, j=1,2, \ldots, l$. Let $t=e^{i \theta} \in S^{1}$ be a generic point. Then

$$
\rho_{j}(t)=\operatorname{diag}\left\{\left(\begin{array}{rr}
\cos x_{j k} \theta & \sin x_{j k} \theta \\
-\sin x_{j k} \theta & \cos x_{j k} \theta
\end{array}\right)\right\},
$$

$k=1,2, \ldots, m / 2$, where each $x_{j k}$ is an integer well defined up to sign.

$$
\tilde{\rho}_{j}(t)=\left[\prod \cos x_{j k} \theta / 2-\sin x_{j k} \theta / 2 e_{2 k-1} e_{2 k}, e^{-i \alpha_{j}}\right]
$$

where $\alpha_{j}=\sum_{k} x_{j k} \theta / 2+\lambda_{j} \theta$ and $\lambda_{j}$ is an integer.

COROLlaRY 5.6. If $S^{1}$ acts on $X$ with l isolated fixed points $p_{j}, j=1,2, \ldots$, $l$, then it is possible to fix the signs of the integers $x_{j k}$ such that the $l \times m / 2$ matrix $\left(\left(x_{j k}\right)\right)$ whose jth row gives the representation of $S^{1}$ on $T X \mid p_{j}$ via (5.4) and the $l$ integers $\lambda_{j}$ determined by (5.5) determine the homomorphism $\operatorname{Id}_{S^{1}}^{X}: K_{S^{1}}^{*}(T X) \rightarrow R\left(S^{1}\right)$ by

$$
\operatorname{Id}_{S^{1}}^{X}\left(\delta_{S^{1}} u\right)=\sum_{j=1}^{l} u_{j}(t) t^{-\lambda_{j}} \prod_{l=1}^{m / 2} \frac{1}{1-t^{x_{j l}}}
$$

where $u_{j}(t) \in R\left(S^{1}\right)=Z\left[t, t^{-1}\right]$ denotes the character obtained by restricting $u$ to $p_{j}$. Moreover if $P$ is the principle $\operatorname{spin}^{c}(m)$ bundle used to define $\delta_{S^{1}}$ and $\omega=P \times{ }_{\operatorname{spin}^{c}(m)} C$ the line bundle determined by $P$, then $\omega$ is an $S^{1}$ bundle over $X$ and the integers $\beta_{j}$ defined by

$$
\left.\omega\right|_{p_{j}}(t)=t^{\beta_{j}} \in Z\left[t, t^{-1}\right]
$$

and the integers $\lambda_{j}$ are related by

$$
-\left(\sum_{k=1}^{m / 2} x_{j k}+2 \lambda_{j}\right)=\beta_{j}
$$

Proof. One of the fundamental properties of the homomorphism $\operatorname{Id}_{G}^{Y}: K_{G}^{*}(T Y) \rightarrow R(G)$ is that for $Y=$ point, it is the identity. By hypothesis

$$
X^{S^{1}}=\bigcup_{j=1}^{l} p_{j}
$$


If $T i_{j}: T p_{j} \rightarrow T X$ is the inclusion,

$$
\begin{aligned}
T i_{j}^{*}\left(\delta_{S^{1}} u\right) & =u_{j}(t) \cdot \prod_{k=1}^{m / 2}\left(t^{x_{j k} / 2}-t^{-x_{j k} / 2}\right) t^{-x_{j k} / 2-\lambda_{j}}, \\
\lambda_{-1}\left(N p_{j} \otimes C\right) & =\prod_{k=1}^{m / 2}\left(1-t^{x_{j k}}\right)\left(1-t^{-x_{j k}}\right) .
\end{aligned}
$$

The signs of the $x_{j k}, k=1,2, \ldots, m / 2$, are chosen as follows: Let

$$
\left.T X\right|_{p_{j}}=e_{1} \oplus e_{2} \oplus \cdots \oplus e_{m / 2}
$$

be the splitting of this representation space of $S^{1}$ into oriented 2 planes invariant under $S^{1}$. The orientation of $\left.T X\right|_{p_{j}}$ is to be the one given by this direct sum representation. Give $e_{i}$ a complex structure compatible with the given orientation on $e_{i}$. Then for $z_{k} \in e_{k}, t \in S^{1}$ operates on $z_{k}$ by multiplication by $t^{x_{j k}}$ and the sign of the integer $x_{j k}$ is determined. Thus

$$
\begin{aligned}
\operatorname{Id}_{S^{1}}^{X}\left(\delta_{S^{1}} u\right) & =\sum_{j} \frac{T i_{j}^{*}\left(\delta_{S^{1}} u\right)}{\lambda_{-1}\left(N p_{j} \otimes C\right)} \text { by (1.4) and (1.6), } \\
& =\sum_{j=1}^{l} u_{j}(t) t^{-\lambda_{j}} \prod_{k=1}^{m / 2}\left(\frac{1}{1-t^{x_{j k}}}\right) .
\end{aligned}
$$

The rest of Corollary 5.6 follows from Proposition 5.3 by replacing the data $\left(\omega_{j}, \theta_{j l}, \lambda_{j}\right)$ by $\left(\beta_{j} \theta, x_{j l} \theta, \lambda_{j} \theta\right)$ and $g$ by $t=e^{i \theta}$.

6. Specialization to $S^{1}$ actions. There is a theorem of Stewart [15] improved by $\mathrm{Su}[16]$ which to my knowledge has found little use until now. It is fundamental to the rest of our discussion. The situation is this: $X$ is a paracompact space supporting a left action of a torus group $\mathfrak{I}_{1}$; $P$ is a principle $\mathfrak{I}_{2}$ bundle over $X$. The torus $\mathfrak{I}_{2}$ acts on the right of $P$.

TheOREM 6.1 (STEWART [15] AND Su [16]). If $H^{1}(X, Z)=0$, the left action of $\mathfrak{I}_{1}$ on $X$ lifts to a left action of $\mathfrak{I}_{1}$ on $P$ which commutes with the principle right action of $\mathfrak{I}_{2}$ on $P$. If $(t, p) \rightarrow t \cdot p$ and $(t, p) \rightarrow t \circ p$ denote two liftings of $\mathfrak{I}_{1}$ to $P$ then there is a homomorphism $\theta: \mathfrak{I}_{1} \rightarrow \mathfrak{I}_{2}$ such that

$$
t \circ p=t \cdot p \cdot \theta(t) \text {. }
$$

We shall restrict application of this theorem to the case $\mathfrak{I}_{1}=\mathfrak{I}_{2}=S^{1}$. Suppose, in addition to the hypothesis of 6.1, that $X$ is a smooth manifold of dimension $m$ and $H^{3}\left(X, Z_{2}\right)=0$. Then $X$ has a $\operatorname{spin}^{c}(m)$ structure by Lemma 3.9. Let $P$ be a principle $\operatorname{spin}^{c}(m)$ bundle over $X$ associated to the tangent bundle of $X$. 
THEOREM 6.2. The left $S^{1}$ action on $X$ lifts to a left $S^{1}$ action on $P$ which commutes with the right action of $\operatorname{spin}^{c}(m)$ on $P$. Thus the action of $X$ satisfies the hypothesis (4.7).

Proof. Let $Q$ denote the principle $S O(m)$ bundle associated to the tangent bundle of $X$. From the exact sequence of groups

$$
S^{1} \rightarrow \operatorname{spin}^{c}(m) \rightarrow S O(m)
$$

we see that $P$ is a principle $S^{1}$ bundle over $Q$. If $m>2$, then $H^{1}(Q, Z)=0$. There is a natural left $S^{1}$ action on $Q$ commuting with the principle right action of $S O(m)$. By 6.1 this action lifts to $P$ and commutes with the principle right action of $S^{1}$.

The lifted action may not commute with the principle $\operatorname{spin}^{c}(m)$ action on $P$ so a modified lifting may be necessary. We have satisfied these.

Hypothesis. $P$ is the total space of a principle right $\operatorname{spin}^{c}(n)$ bundle over $X$. There is a left action of $S^{1}$ on $P$ which commutes with the right action of $S^{1} \subset \operatorname{spin}^{c}(n)$. Moreover, the induced left action of $S^{1}$ on the orbit space $Q=P / S^{1}$ commutes with the right action of $S O(n)$ on $Q$.

We now show that this hypothesis implies that the left action of $S^{1}$ on $P$ can be modified so as to commute with the principle right action of $\operatorname{spin}^{c}(n)$ and so that the induced left action on $Q$ is left unaltered.

Let $s \in S^{1}, h \in \operatorname{spin}^{c}(n)$. Then for $x \in P$ we have

(i) $(s x) h=s(x h) \hat{\psi}(s, x, h)$ where $\hat{\psi}(s, x, h) \in S^{1}$, the center of $\operatorname{spin}^{c}(n)$. This is a consequence of the fact that the $S^{1}$ action on $Q$ commutes with the right action of $S O(n)$.

(ii) $\hat{\psi}(s, x t, h)=\hat{\psi}(s, x, h)$ for $t \in S^{1}$. This is a consequence of the fact that $t \in$ center $\operatorname{spin}^{c}(n)$ and the Hypothesis.

As a result of (ii), $\hat{\psi}(s, x, h)=(s, \pi x, h)$ where $\psi: S^{1} \times Q \times S O(n) \rightarrow S^{1}$. Here $\pi$ is the projection of $P$ on $Q$. The function $\psi$ has these properties:

(iii) $\psi(1, z, h)=\psi(s, z, 1)=1, z \in Q$ and 1 the identity of the appropriate group.

(iv) $\psi\left(s_{1} s_{2}, z, h\right)=\psi\left(s_{1}, s_{2} z, h\right) \psi\left(s_{2}, z, h\right)$.

(v) $\psi\left(s, z, h_{1} h_{2}\right)=\psi\left(s, z h_{1}, h_{2}\right) \psi\left(s, z, h_{1}\right)$.

Because of (iii), $\psi$ is null homotopic and there is a unique lifting $\widetilde{\psi}$ to $R^{1}$ which satisfies $\widetilde{\psi}\left(1, z_{0}, 1\right)=1$ for a fixed $z_{0} \in Q$. Moreover $\widetilde{\psi}$ will satisfy (iii), (iv) and (v) except we change from a multiplicative to an additive notation. Observe that

(vi) $\psi\left(s, z h^{\prime}, h^{\prime-1} h\right)-\psi\left(s, z h^{\prime}, h^{\prime-1}\right)=\widetilde{\psi}(s, z, h)$ by (v).

Define, for $s \in S^{1}, z \in Q$,

$$
\gamma(s, z)=\int_{H} \widetilde{\psi}\left(s, z h, h^{-1}\right) d h
$$


where $d h$ denotes the normalized Haar measure on $\operatorname{spin}^{c}(n)=H$. Then

$$
\begin{aligned}
\gamma(s, z h)-\gamma(s, h) & =\int_{H} \widetilde{\psi}\left(s, z h h^{\prime}, h^{\prime-1}\right) d h^{\prime}-\int_{H} \widetilde{\psi}\left(s, z h^{\prime}, h^{\prime-1}\right) d h^{\prime} \\
& =\int_{H}\left[\widetilde{\psi}\left(s, z h^{\prime \prime}, h^{\prime \prime}-1\right)-\widetilde{\psi}\left(s, z h^{\prime \prime}, h^{\prime \prime}-1\right)\right] d h^{\prime \prime} \\
& =\int_{H} \widetilde{\psi}(s, z, h) d h^{\prime \prime}=\widetilde{\psi}(s, z, h) \quad \text { by }(\mathrm{vi}) .
\end{aligned}
$$

(viii) $\gamma\left(s_{1} s_{2}, z\right)=\gamma\left(s_{1}, s_{2} z\right)+\gamma\left(s_{2}, z\right)$ by (iv).

Let $\bar{\gamma}(s, z) \in S^{1}$ denote the image in $S^{1}$ of $\gamma(s, z)$ under the covering map and define a new left action of $S^{1}$ on $P$ by

$$
s \circ x=s x(\bar{\gamma}(s, z(x)))
$$

where $z: P \rightarrow Q$ is the projection. Then

$$
\begin{aligned}
& (s \circ x) h=(s x \bar{\gamma}(s, z(x)) h)=s(x h) \psi(s, z(x), h) \cdot \bar{\gamma}(s, z(x)), \\
& s \circ(x h)=s(x h) \bar{\gamma}(s, z(x), h) .
\end{aligned}
$$

Since $\bar{\gamma}(s, z(x) h) \bar{\gamma}(s, z(x))^{-1}=\psi(s, z(x), h)$ by (vii) we have

(ix) $(s \circ x) h=s \circ(x h)$;

(x) $\left(s_{1} s_{2}\right) \circ x=s_{1} \circ\left(s_{2} \circ x\right)$ by (viii).

Property (x) shows that $\circ$ is an action and property (ix) shows that this left action of $S^{1}$ commutes with the right action of $\operatorname{spin}^{c}(n)$. It follows from the definition of o that the induced left action of $S^{1}$ on $Q$ agrees with the original action.

COROLlaRY 6.3. Let $X$ be a smooth manifold with $H^{1}(X, Z)=0$ and which supports a smooth $S^{1}$ action. Then the class $\delta_{S^{1}} \in K_{S^{1}}^{*}(T X)$ is defined and is an orientation for $X$.

Proof. This follows from Theorem 6.2 and Proposition 4.9.

6.4. Standard example. Here is an important example which illustrates the foregoing remarks: Let $X=C P^{n}=U(n+1) / U(1) \times U(n) . X$ is a $\operatorname{spin}^{c}(2 n)$ manifold. A principle $\operatorname{spin}^{c}(2 n)$ bundle associated to $T C P^{n}$ has total space

$$
U(n+1) \times_{H} \operatorname{spin}^{c}(2 n)=P
$$

where $H=U(1) \times U(n)$ acts on $\operatorname{spin}^{c}(2 n)$ through the composition (see (2.3))

$$
H \stackrel{\mathrm{Ad}}{\longrightarrow} U(n) \stackrel{\psi_{0}}{\longrightarrow} \operatorname{spin}^{c}(2 n) .
$$


For $x \in U(1)=S^{1}$, let $d(x) \in U(n)$ be the diagonal matrix with $d_{i i}(x)=x$. Then $\operatorname{Ad}(x, y)=d\left(x^{-1}\right) y$ for $y \in U(n)$. Moreover, the complex line bundle

$$
\omega=P \times_{\operatorname{spin}^{c}(2 n)} C=U(n+1) \times{ }_{H} C
$$

is $\mathscr{H}^{n+1}$ where $\mathscr{H}$ is the Hopf bundle over $X$.

Left action of $U(n+1)$ on the coset space $U(n+1) / H$ makes $X$ into a left $U(n+1)$ space. Any representation $\phi: S^{1} \rightarrow U(n+1)$ will then define an action of $S^{1}$ on $X$. Such a representation is given by

$$
\phi(t)=\left[\begin{array}{ccc}
t^{a_{0}} & & \\
& t^{a_{1}} & \\
& & \ddots \\
& & t^{a_{n}}
\end{array}\right] \subset U(n+1)
$$

for $t \in S^{1}$ and integers $a_{i}$. In terms of homogenous coordinates $\left(z_{0}: z_{1}: \ldots: z_{n}\right)$ on $C P^{n}$, the action takes the form

$$
t\left(z_{0}: z_{1}: \ldots: z_{n}\right)=\left(t^{a_{0}} z_{0}: t^{a_{n}} z_{1}: \ldots: t^{a_{n}} z_{n}\right)
$$

and if the integers $a_{j}$ are distinct, there are $n+1$ isolated fixed points

$$
p_{i}=(0: 0: \ldots: 1: \ldots: 0)
$$

all of whose coordinates are zero except the $i$ th which is 1 . The representation $\rho_{j}$ of $S^{1}$ on $\left.T X\right|_{p_{j}}$ is given by

$$
\rho_{j}\left(e^{i \theta}\right)=\operatorname{diag}\left\{\left(\begin{array}{rr}
\cos \left(a_{k}-a_{j}\right) \theta & \sin \left(a_{k}-a_{j}\right) \theta \\
-\sin \left(a_{k}-a_{j}\right) \theta & \cos \left(a_{k}-a_{j}\right) \theta
\end{array}\right)\right\} \subset S O(2 n), \quad k \neq j .
$$

The $S^{1}$ action is already lifted to the principle $\operatorname{spin}^{c}(2 n)$ bundle $P$. Explicitly

$$
t[u, s]=[\phi(t) u, s]
$$

for $t \in S^{1}, u \in U(n+1)$ and $s \in \operatorname{spin}^{c}(2 n)$. Moreover

$$
\begin{aligned}
\tilde{\rho}_{j}\left(e^{i \theta}\right)=\prod_{k \neq j}\left[\cos \left(a_{k}-a_{j}\right) \theta / 2\right. & \\
& \left.-\sin \left(a_{k}-a_{j}\right) \theta / 2 e_{2 k-1} e_{2 k}, \exp \left[-i \sum\left(a_{k}-a_{j}\right) \theta / 2\right]\right] .
\end{aligned}
$$

The Hopf bundle $\mathscr{H}=U(n+1) \times{ }_{H} C$, where $H$ acts on $C$ via the representation $U(1) \times U(n) \rightarrow U(1)$ defined by $(x, y) \rightarrow x$, becomes an $S^{1}$ bundle over $X$ by setting

$$
t \circ[u, c]=[\phi(t) u, c], \quad u \in U(n+1), c \in C .
$$


In the same manner the bundle $\mathscr{H}^{n+1}$ becomes an $S^{1}$ bundle over $X$ and, by (iii),

$$
\left.\mathscr{H}\right|_{p_{j}}(t)=t^{a_{j}},\left.\quad \mathscr{H}^{n+1}\right|_{p_{j}}(t)=t^{(n+1) a_{j}} \in Z\left[t, t^{-1}\right] .
$$

Since $\omega$ and $\mathscr{H}^{n+1}$ are isomorphic as vector bundles, their associated principle $S^{1}$ bundles are isomorphic and since we have two liftings of $S^{1}$ to this principle bundle $P(\omega)=P\left(\mathscr{H}^{n+1}\right)$, by 6.1 there is an integer $\theta$ such that

$$
t \circ p=t \cdot p \cdot t^{\theta}, \quad t \in S^{1}, p \in P(\omega)=P\left(\mathscr{H}^{n+1}\right) .
$$

This integer is determined by the restriction of $\omega$ and $\mathscr{H}^{n+1}$ to any fixed point $p_{j}$. But

$$
\left.\omega\right|_{p_{j}}(t)=t^{-\Sigma_{k \neq j}\left(a_{k}-a_{j}\right)} \quad \text { by (i) and (ii), }
$$

while

$$
\left.\mathscr{H}^{n+1}\right|_{p_{j}}(t)=t^{(n+1) a_{j}}
$$

so

$$
\theta-\sum_{k \neq j}\left(a_{k}-a_{j}\right)=(n+1) a_{j}
$$

and

$$
\theta=\sum_{k=0}^{n} a_{k}
$$

The character $\operatorname{Id}_{S^{1}}^{C P^{n}}\left(\mathscr{H}^{k} \delta_{S^{1}}\right)$ is given by

$$
\sum_{j=0}^{n} t^{k a_{j}} \prod_{l \neq j}\left(1-t^{\left(a_{l}-a_{j}\right)}\right)^{-1}
$$

by Corollary $5.6 \quad\left(\lambda_{j}=0\right.$ by (5.5) and (ii)).

As a second application of the Stewart theorem we define a homomorphism $F$ from the additive group $H^{2}(X, Z)$ to the multiplicative group of units of $K_{S^{1}}^{*}(X)$. We assume $H^{2}(X, Z)$ is free abelian. Let $z_{1}, \ldots, z_{s}$ be a basis for this group. The $z_{i}$ 's determine complex line bundles over $X$ which we also denote by $z_{i}$. Let $P_{i}$ denote the principle $S^{1}$ bundle over $X$ associated to $z_{i}$. Then

$$
z_{i}=P_{i} \times{ }_{S^{1}} C .
$$

By 6.1, the left $S^{1}$ action on $X$ lifts to a left $S^{1}$ action on $P_{i}$ which commutes with the right principle $S^{1}$ action on $P_{i}$. Define a left $S^{1}$ action on $z_{i}$ by

$$
t[p, c]=[t p, c] .
$$

Then $z_{i}$ with this action of $S^{1}$ becomes an element $F\left(z_{i}\right)=W_{i} \in K_{S^{1}}^{*}(X)$. 
Define $F\left(\sum \lambda_{j} z_{j}\right)=\prod W_{j}^{\lambda_{j}}, \lambda_{j} \in Z$.

Let $L\left(z_{1}, \ldots, z_{s}\right)=\sum n_{j} z_{j}=L, n_{j} \in Z$, be an element whose $\bmod 2$ reduction is $W_{2}(X)$ and $P_{L}$ the principle $\operatorname{spin}^{c}(m)$ bundle over $X$ defined by $L$, i.e., $P_{L} \times_{\text {spinc }^{\prime}(m)} C$ is the line bundle with the first Chern class $L$. Use Theorem 6.1 to lift the $S^{1}$ action to $P_{L}$. Suppose for simplicity that $X^{S^{1}}$ consists of $n$ isolated fixed points $\left\{p_{i}\right\}$ and define integers $z_{i j}, i=1, \ldots, s$, $j=1,2, \ldots, n$, by

$$
\left.W_{i}\right|_{p_{j}}(t)=t^{z_{i j}} \in Z\left[t, t^{-1}\right]
$$

and integers $x_{i j}$ (defined up to sign) by

$$
\rho_{i}(t)=\operatorname{diag}\left(\begin{array}{rr}
\cos x_{i j} \theta & \sin x_{i j} \theta \\
-\sin x_{i j} \theta & \cos x_{i j} \theta
\end{array}\right), \quad j=1,2, \ldots, m / 2, i=1,2, \ldots, n .
$$

Then set $L_{i}=L\left(z_{1 i}, z_{2 i}, \ldots, z_{s i}\right)=\sum n_{k} z_{k i} \in Z$.

THEOREM 6.5. There is an integer $N$ such that for any polynomial $\Phi\left(y_{1}, y_{2}, \ldots, y_{s}\right)$ with integer coefficients,

$$
\begin{aligned}
E_{\Phi}= & t^{N / 2} \sum_{i=1}^{n} \Phi\left(t^{z_{1 i}}, t^{z_{2 i}}, \ldots, t^{z_{s i}}\right) \\
& \cdot t^{L_{i} / 2} \prod_{j=1}^{m / 2}\left(t^{-x_{i j} / 2}-t^{x_{i j} / 2}\right)^{-1} \in Z\left[t, t^{-1}\right]=R\left(S^{1}\right) .
\end{aligned}
$$

Proof. Since $\prod W_{i}^{n_{i}}$ and $P_{L} \times_{\operatorname{spin}^{c}(m)} C=\omega$ are isomorphic as vector bundles there is an integer $N$ such that

$$
\begin{aligned}
\left.\omega\right|_{p_{j}}(t) & =\left.t^{N} \cdot \prod_{i} W_{i}^{n_{i}}\right|_{p_{j}}(t) \quad(\text { by 6.1) } \\
& =t^{N} \cdot \prod_{i} t^{n_{i z_{i j}}}=t^{N+L_{j}} .
\end{aligned}
$$

The result follows from Corollary 5.6 by setting $\beta_{j}=N+L_{j}$ and $\mu_{i}=\Phi\left(t^{z_{1 i}}, t^{z_{2 i}}, \ldots, t^{z_{s i}}\right)$ because $E_{\Phi}=\operatorname{Id}_{S^{1}}^{X}\left(\Phi\left(W_{1}, W_{2}, \ldots, W_{s}\right) \delta_{S^{1}}\right)$ and

$$
\begin{aligned}
t^{\left(N+L_{i}\right) / 2} \cdot \prod_{j=1}^{m / 2}\left(t^{-x_{i j} / 2}-t^{x^{i j} / 2}\right)^{-1} & =t^{-\lambda_{i}} \prod_{j=1}^{m / 2}\left(1-t^{x_{i j}}\right)^{-1}, \\
\lambda_{j} & =\left(\sum x_{i j} / 2+\beta_{j} / 2\right) .
\end{aligned}
$$

REMARK. Because of the occurrence of square roots in the terms in the expression for $E_{\Phi}$, these terms are only defined up to sign. We will have more to say later. 
II. AppliCATIONS to $S^{1}$ ACTIONS ON HOMOTOPY COMPLEX PROJECTIVE SPACES

Part II of the paper is devoted to studying this situation: $X$ is a smooth manifold homotopy equivalent to $C P^{n}$ and supports a smooth $S^{1}$ action. What properties of the example of Part I, (6.4) persist here?

1. Generalities. The most important property of this situation is

Proposition 1.1. Let $\eta^{\prime}$ be the pull back of the Hopf bundle over $C P^{n}$ via a homotopy equivalence from $X$ to $C P^{n}$. Then $\eta^{\prime}$ admits an $S^{1}$ action making it an $S^{1}$ bundle $\eta$ over $X$.

Proof. Let $F: H^{2}(X, Z) \rightarrow K_{S^{1}}^{*}(X)$ be the function defined in $\S 6$. Then $\eta=F\left[c_{1}\left(\eta^{\prime}\right)\right]$ where $c_{1}\left(\eta^{\prime}\right)$ is the first Chern class of $\eta^{\prime}$. It generates $H^{2}(X, Z)$.

Let $X^{S^{1}}$ denote the fixed point set of $S^{1}$ acting on $X$. Then

$$
X^{S^{1}}=X_{0} \cup X_{1} \cup \cdots \cup X_{l-1}
$$

is the disjoint union of $l$ connected components $X_{i}$ and each $X_{i}$ is a cohomology $C P^{k_{i}-1}$ where $\sum_{i=0}^{l-1} k_{i}=n+1$. Moreover, the natural map $H^{*}(X) \rightarrow H^{*}\left(X_{i}\right)$ is an isomorphism for $* \leqq 2 k_{i}+1$. This is a result of [9], [11].

Choose a point $p_{j} \in X_{j}$.

DEFINITION 1.2. Define l integers $a_{j}$ by $\left.\eta\right|_{p_{j}}(t)=t^{a_{j}}$.

REMARK. The integers $a_{j}$ are not uniquely defined by the $S^{1}$ action on $X$; they depend on the lifting of the action to the principle $S^{1}$ bundle associated to $\eta$. However a second lifting will give rise to a new set of integers $a_{j}^{\prime}$ which are related to the old set by

$$
a_{j}^{\prime}=a_{j}+\theta \text {; }
$$

This is a consequence of Part I, 6.1. Thus the integers $a_{j}$ are well defined up to translation.

Having this set of integers, we can now compare the given action with the $S^{1}$ action on $C P^{n}$ of 6.3 which is determined by the representation $\phi: S^{1} \rightarrow U(n+1)$ defined by

$$
\phi(t)=\operatorname{diag}\left\{t^{-a_{j}} I_{j}\right\}_{j=0,1, \ldots, l-1} .
$$

$I_{j}$ is the identity matrix of dimension $k_{j}$.

REMARK. The complex $K$ theory of $X$ and of the components $X_{i}$ of $X^{S^{1}}$ is given by

$$
\begin{aligned}
& K^{*}(X) \cong Z\left[\eta^{\prime}\right] /\left(\left(\eta^{\prime}-1\right)^{n+1}\right), \\
& K^{*}\left(X_{i}\right) \cong Z\left[\eta_{i}^{\prime}\right] /\left(\left(\eta_{i}^{\prime}-1\right)^{k_{i}}\right),
\end{aligned}
$$

where $\eta_{i}^{\prime}$ is the restriction of $\eta^{\prime}$ to $X_{i}$. 
The homomorphism $\tau: 1 \rightarrow S^{1}$ of the trivial group to $S^{1}$ induces a homomorphism $\tau^{*}: K_{S^{1}}^{*}(X) \rightarrow K^{*}(X)$.

LEMMA 1.3. $\tau^{*}$ is surjective.

Proof. Since $\tau^{*} \eta=\eta^{\prime}, \tau^{*}$ is surjective by the above Remark.

LEMMA 1.4. There is an exact sequence

$$
0 \rightarrow K_{S^{1}}^{*}(X) \stackrel{\Delta}{\longrightarrow} K_{S^{1}}^{*}(X) \stackrel{\tau^{*}}{\longrightarrow} K^{*}(X) \rightarrow 0
$$

where $\Delta$ is multiplication by $(t-1) \in Z\left[t, t^{-1}\right]$.

Proof. Let $S^{1}$ act on $\left(X \times D^{2}, X \times S^{1}\right)$ by $t(x, d)=(t x, t d)$ for $x \in X$, $d \in D^{2}$ and $t \in S^{1}$. Then on $X \times S^{1}$ this action is equivalent to the action defined by

$$
t(x, d)=(x, t d), \quad\|d\|=1 .
$$

An equivalence of actions is provided by

$$
(x, d) \rightarrow\left(d^{-1} x, d\right), \quad\|d\|=1 .
$$

In view of this,

$$
K_{S^{1}}^{*}\left(X \times S^{1}\right) \cong K^{*}\left(X \times S^{1} / S^{1}\right) \cong K^{*}(X)
$$

because $S^{1}$ acts freely on $X \times S^{1}$. By the Thom isomorphism theorem [1],

$$
K_{S^{1}}^{*}(X) \cong K_{S^{1}}^{*}\left(X \times D^{2}, X \times S^{1}\right) .
$$

This isomorphism composed with the restriction

$$
K_{S^{1}}^{*}\left(X \times D^{2}, X \times S^{1}\right) \rightarrow K_{S^{1}}^{*}\left(X \times D^{2}\right) \cong K_{S^{1}}^{*}(X)
$$

is multiplication by $(t-1)$ [1]. Making these identifications, the exact triangle for the pair $\left(X \times D^{2}, X \times S^{1}\right)$ becomes

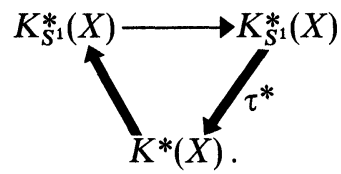

Since $\tau^{*}$ is surjective, the result follows.

Let $\mathfrak{I} \subset K_{S^{1}}^{*}(X)$ be the $\Lambda=Z\left[t, t^{-1}\right]$ torsion subgroup of $K_{S^{1}}^{*}(X)$.

LEMMA 1.5. $\tau^{*}$ maps $\mathfrak{I}$ to zero.

Proof. By Lemma 1.4, $\mathfrak{I}$ has no $t-1$ torsion. Let $x \in \mathfrak{I}$ and $\lambda \in \Lambda$ such that $\lambda x=0$. We may suppose that $\lambda$ is prime to $t-1$. Then in $\Lambda \otimes Q$ there are elements $a$ and $b$ such that

$$
1=a \lambda+b(t-1) \text {. }
$$


Let $d$ be an integer such that $d a=a^{\prime}$ and $d b=b^{\prime}$ are in $\Lambda$. Then $d=a^{\prime} \lambda+b^{\prime}(t-1)$ and $d x=b^{\prime}(t-1) x$ so

$$
d \tau^{*}(x)=\tau^{*}(d x)=\tau^{*}\left(b^{\prime}(t-1) x\right)=0
$$

by Lemma 1.4. Since $K^{*}(X)$ is free abelian, $\tau^{*}(x)=0$.

Lemma 1.6. Let $i: X^{S^{1}} \rightarrow X$ denote the inclusion of the fixed point set. Then $i^{*}$ maps $\mathfrak{I}$ to zero.

Proof. $K_{S^{1}}^{*}\left(X^{S^{1}}\right)=\prod_{i=0}^{l-1} K^{*}\left(X_{i}\right) \otimes_{Z} \Lambda$ is a free $\Lambda$ module by the Remark above.

Let $\hat{K}_{S^{1}}(X)=K_{S^{1}}^{*}(X) / \mathfrak{I}$. Then the above two lemmas imply that we may regard the domain of $\tau^{*}$ and $i^{*}$ as $\hat{K}_{S^{1}}(X)$.

Define $f(x) \in \Lambda[x]$ by

$$
f(x)=\prod_{i=0}^{l=1}\left(x-t^{a_{i}}\right)^{k_{i}}
$$

and define a map of algebras $A^{\prime}$ from $\Lambda[x]$ to $\hat{K}_{S^{1}}(X)$ by $A^{\prime}(x)=\eta$.

Lemma 1.8. $A^{\prime}(f(x))=0$.

ProOF. Let $\mathfrak{p} \in \Lambda$ be the prime ideal of characters which vanish at a generic $t \in S^{1}$. Then $X^{t}=\{x \in X \mid t x=x\}=X^{S^{1}}$. Let $\Lambda_{\mathfrak{p}}$ be $\Lambda$ localized at $\mathfrak{p}$. By the Atiyah-Segal localization theorem Part I, (1.6), $i_{\mathfrak{p}}^{*}: \hat{K}_{S^{1}}(X)_{\mathfrak{p}}$ $\rightarrow K_{S^{1}}^{*}\left(X^{t}\right)_{\mathfrak{p}}$ is an isomorphism. Since $\hat{K}_{S^{1}}(X)$ is a torsion free $\Lambda$ module and $K_{S^{1}}^{*}\left(X^{t}\right)$ is a free $\Lambda$ module, this means that $i^{*}$ is a monomorphism. But $i^{*} A^{\prime}(f(x))=0$ so $A^{\prime}(f(x))=0$.

Thus there is an induced map of algebras

$$
A: \Lambda[x] /(f(x)) \rightarrow \hat{K}_{S^{1}}(X) .
$$

Let $p \in Z$ be a prime and $\xi$ a primitive $p^{\text {th }}$ root of unity, $Q(\xi)=k$ the field of primitive $p^{\text {th }}$ roots of unity and $\mathcal{O}$ the integers in $k$. Then $\mathcal{O}$ is a $\Lambda$ module via $g(t) \rightarrow g(\xi)$ when $g(t) \in \Lambda$. Clearly if $\Gamma=\Lambda[x] /(f(x))$, then

$$
\Gamma \otimes_{\Lambda} \mathcal{O}=\mathcal{O}[x] /(\bar{f}(x)) \quad \text { where } \bar{f}(x)=\prod_{a \in Z_{p^{r}}}\left(x-\xi^{a}\right)^{d_{a}} ;
$$

$d_{a}$ is the sum of the $k_{j}$ with $a_{j} \equiv a\left(p^{r}\right)$. Moreover

$$
\Gamma \otimes_{\Lambda} k \cong \prod_{a \in Z_{p r}} k[x] /\left(\left(x-\xi^{a}\right)^{d_{a}}\right)
$$

as an algebra.

We shall see that $\hat{K}_{S^{1}}(X) \otimes_{\Lambda} k$ is a useful tool for studying the fixed point set $X^{Z_{p r}}$.

LEMMA 1.9. $A \otimes_{\Lambda} 1_{k}: \Gamma \otimes_{\Lambda} k \rightarrow \hat{K}_{S^{1}}(X) \otimes_{\Lambda} k$ is an isomorphism of algebras. 
Proof. The $\Lambda$ rank of $\hat{K}_{S^{1}}(X)$ is $n+1$ because $K_{S^{1}}^{*}\left(X^{S^{1}}\right)=$ $R\left(S^{1}\right) \otimes K^{*}\left(X^{S^{1}}\right)$ is a free $\Lambda$ module of rank $n+1$ and $K_{S^{1}}^{*}(X)$ has the same $\Lambda$ rank as $K_{S^{1}}^{*}\left(X^{S^{1}}\right)$ by Part I, (1.6). It suffices to show that $A$ induces a monomorphism

$$
A=A \otimes_{\Lambda} 1_{\mathscr{O}}: \Gamma \otimes_{\Lambda} \mathcal{O} \rightarrow \hat{K}_{S^{1}}(X) \otimes_{\Lambda} \mathcal{O} .
$$

It is easy to check that the kernel of the composition

$$
\Gamma \otimes_{\Lambda} \mathcal{O} \rightarrow \hat{K}_{S^{1}}(X) \otimes_{\Lambda} \mathcal{O} \stackrel{\tau^{*} \otimes_{\Lambda} 1}{\longrightarrow} K^{*}(X) \otimes_{\Lambda} \mathcal{O} \cong K(X) \otimes \mathcal{O} /(1-\xi) \mathcal{O}
$$

is $\Gamma \otimes_{\Lambda}(1-\xi) \mathcal{O}$. This means that if $x$ is in the kernel of $\tilde{A}, x=(1-\xi) x_{1}$. Since $\hat{K}_{S^{1}}(X)$ is torsion free, $\tilde{A}\left(x_{1}\right)=0$ and inductively $x=(1-\xi)^{n} x_{n}$. Since $\Gamma \otimes_{\Lambda} \mathcal{O}$ is a free $\mathcal{O}$ module and $(1-\xi)$ is not a unit of $\mathcal{O}$, this can only happen if $x$ is zero.

Let $\mathfrak{T}_{p^{r}}$ be the $\Lambda$ torsion subgroup of $K_{S^{1}}^{*}\left(X^{Z_{p^{r}}}\right)$ and let $j$ denote the inclusion of $X^{Z_{p}^{r}}$ in $X$.

Lemma 1.10. The composition

$$
\Gamma \otimes_{\Lambda} k \stackrel{A \otimes_{\Lambda} 1}{\longrightarrow} \hat{K}_{S^{1}}(X) \otimes_{\Lambda} k \stackrel{j^{*} \otimes_{\Lambda} 1}{\longrightarrow} \hat{K}_{S^{1}}\left(X^{Z_{p^{r}}}\right) \otimes_{\Lambda} k
$$

is an isomorphism.

PRoOF. Let $\mathfrak{p}$ be the prime ideal of characters vanishing at $\xi \in S^{1}$. Then $j_{\mathfrak{p}}^{*}: K_{S^{1}}(X)_{\mathfrak{p}} \rightarrow K_{S^{1}}\left(X^{Z_{p} r}\right)_{\mathfrak{p}}$ is an isomorphism. This implies that $j_{\mathfrak{p}}^{*}: \mathfrak{T}_{\mathfrak{p}}$ $\rightarrow\left(\mathfrak{I}_{p^{r}}\right)_{p}$ is an isomorphism; hence, $j_{\mathfrak{p}}^{*}$ induces an isomorphism $\hat{K}_{S^{1}}(X)_{\mathfrak{p}}$ $\rightarrow \hat{K}_{S^{1}}\left(X^{Z_{p^{r}}}\right)_{\mathfrak{p}}$. Tensoring these groups over $\Lambda_{\mathfrak{p}}$ with $k$ and observing that

$$
\begin{aligned}
\hat{K}_{S^{1}}(X) \otimes_{\Lambda_{p}} \dot{k} & =\hat{K}_{S^{1}}(X) \otimes_{\Lambda} k, \\
\hat{K}_{S^{1}}\left(X^{Z_{p^{r}}}\right) \otimes_{\Lambda_{\mathfrak{p}}} k & =\hat{K}_{S^{1}}\left(X^{\left.Z_{p^{r}}\right) \otimes_{\Lambda} k,}\right.
\end{aligned}
$$

we obtain the desired result.

Let $Z_{\infty}$ be the union of those components of $X^{Z_{p r}}$ which miss $X^{S^{1}}$ and let $Z_{a}, a \in Z_{p^{r}}$, be the union of those components which contain an $X_{i}$ with $a_{i} \equiv a\left(p^{r}\right)$.

LEMMA 1.11. $Z_{a}$ is connected and empty if there is no $a_{i} \equiv a\left(p^{r}\right)$.

Proof. Since there are no points of $Z_{\infty}$ fixed by $S^{1}$ by definition, it follows from the Atiyah-Segal localization theorem that $K_{S^{1}}^{*}\left(Z_{\infty}\right)$ is a $\Lambda$ torsion module. Moreover

$$
K_{S^{1}}^{*}\left(X^{Z_{p^{r}}}\right)=\prod_{a \in Z_{p r}} K_{S^{1}}^{*}\left(Z_{a}\right) \times K_{S^{1}}^{*}\left(Z_{\infty}\right)
$$

and the torsion subgroup $\mathfrak{I}_{p^{r}}$ splits as

$$
\mathfrak{I}_{p^{r}}=\prod_{a} \mathfrak{I}_{a} \times K_{S^{1}}^{*}\left(Z_{\infty}\right)
$$


where $\mathfrak{I}_{a}$ is the torsion subgroup of $K_{S^{1}}^{*}\left(Z_{a}\right)$. Thus

$$
\hat{K}_{S^{1}}\left(X^{Z_{p^{r}}}\right) \cong \prod_{a} \hat{K}_{S^{1}}\left(Z_{a}\right) \text {. }
$$

Suppose $Z_{a} \neq \varnothing$. Then for some $i$ there are $S^{1}$ equivariant maps (see Definition 1.2)

$$
p_{i} \rightarrow Z_{a} \rightarrow p_{i}
$$

which imply that $\Lambda=K_{S^{1}}^{*}\left(p_{i}\right)$ is a direct factor of $\hat{K}_{S^{1}}\left(Z_{a}\right)$ so $\hat{K}_{S^{1}}\left(Z_{a}\right)$ is nonzero. By Lemma 1.10 we have

$$
\prod_{a} k[x] /\left(\left(x-\xi^{a}\right)^{d_{a}}\right) \cong \prod_{a} \hat{K}_{S^{1}}\left(Z_{a}\right) \otimes k .
$$

This is an isomorphism of $k[x]$ modules; so by the unique decomposition theorem for torsion modules over $k[x]$, [14],

$$
\hat{K}_{S^{1}}\left(Z_{a}\right) \otimes_{\Lambda} k \cong \prod_{a \in S_{a}} k[x] /\left(\left(x-\xi^{a}\right)^{d_{a}}\right)
$$

where $S_{a}$ is some subset of $Z_{p^{r}}$ which is nonzero if $Z_{a} \neq \varnothing$.

Since the number of factors on the left of (1.12) is precisely the number of distinct residue classes appearing among the $\left\{a_{i}\right\}$ and since the number of factors occurring on the right side of (1.12) is at least this number, it follows that $S_{a}$ is zero if there is no $a_{i} \equiv a\left(p^{r}\right)$ and just $a$ if there is an $a_{i} \equiv a\left(p^{r}\right)$.

Suppose some $Z_{a} \neq \varnothing$ is not connected. Then $Z_{a}=Y_{1} \cup Y_{2}$ where $Y_{1}$ and $Y_{2}$ contain points $p_{i}$ and $p_{j}$ with $a_{i} \equiv a \equiv a_{j}$ so $\hat{K}_{S^{1}}\left(Y_{i}\right) \neq 0, i=1,2$, and

$$
\hat{K}_{S^{1}}\left(Z_{a}\right) \cong \hat{K}_{S^{1}}\left(Y_{1}\right) \times \hat{K}_{S^{1}}\left(Y_{2}\right) .
$$

But this implies that there are more nonzero summands on the right side of (1.12) than on the left.

COROLlaRY 1.13 .

$$
\hat{K}_{S^{1}}\left(X^{Z_{p^{r}}}\right) \otimes_{\Lambda} k \cong \prod_{a \in Z_{p^{r}}} k[x] /\left(\left(1-\xi^{a}\right)^{d a}\right)
$$

as an algebra.

COROLlary 1.14. A sufficient condition that the fixed point set of $Z_{p^{r}}$ strictly contain the fixed set of $S^{1}, X^{S^{1}}$, is that there are a pair of distinct integers $i$ and $j$ with $a_{i} \equiv a_{j}\left(p^{r}\right)$.

ProOF. We have seen in the proof of Lemma 1.11, that the number of connected components of $X^{Z_{p r}}$ intersecting $X^{S^{1}}$ is the number of distinct residue classes among the $l$ integers $a_{i}$, while the number of components of $X^{S^{1}}$ is $l$ by assumption. If the number of distinct residue classes among 
the $a_{j}$ is less than $l$, then two components of $X^{S^{1}}$ are in the same component of $X^{Z_{p r}}$ showing $X^{Z_{p r} r}$ strictly contains $X^{S^{1}}$.

COROLlaRY 1.15. The lintegers $a_{i}$ are distinct.

Proof. For $r$ large, $X^{Z_{p r}}=X^{S^{1}}$ for any prime $p$. If some pair $\left(a_{i}, a_{j}\right)$ were equal, then the collection of $l$ integers $\left\{a_{j}\right\}$ would contain fewer than $l$ residue classes $\bmod p^{r}$ so $X^{Z_{p r}}$ would strictly contain $X^{S^{1}}$ by the preceding corollary.

2. Isolated fixed points. Now we restrict our attention to the case in which $X^{S^{1}}$ consists of isolated fixed points. Then there must be $n+1$ such points $p_{j}, j=0,1,2, \ldots, n$. To each is attached an integer $a_{j}$ as discussed in $\S 1$ and the $n+1$ integers $a_{j}$ are distinct by Corollary 1.15. Our aim is to compare the given action of $S^{1}$ on $X$ with the linear action of $S^{1}$ on $C P^{n}$ defined by the integers $a_{i}$ of Part I, 6.4.

Introduce an $(n+1) \times n$ matrix of integers $x_{i j}, i=0,1,2, \ldots, n$, $j=1,2, \ldots, n$ (defined up to sign), whose $i$ th row gives the representation of $S^{1}$ on $\left.T X\right|_{p_{i}}$ by

$$
\rho_{i}(t)=\operatorname{diag}\left\{\left(\begin{array}{rr}
\cos x_{i j} \theta & \sin x_{i j} \theta \\
-\sin x_{i j} \theta & \cos x_{i j} \theta
\end{array}\right)\right\}, \quad j=1,2, \ldots, n, t=e^{i \theta} .
$$

LeMMA 2.1.

$$
\psi_{i}(t)=\prod_{j \neq i}\left(1-t^{a_{j}-a_{i}}\right) \cdot \prod_{j=1}^{n}\left(1-t^{x_{i j}}\right)^{-1} \in \Lambda .
$$

PROOF. Let $e_{i}=\prod_{j \neq i}\left(\eta-t^{a_{j}}\right) \in \hat{K}_{S^{1}}(X)$. Then $\delta_{S^{1}} e_{i} \in \hat{K}_{S^{1}}(T X)$ and

$$
\operatorname{Id}_{S^{1}}^{X}\left(\delta_{S^{1}} e_{i}\right) \in \Lambda \text {. }
$$

Since the character of $\left.\eta\right|_{p_{j}}$ is $t^{a_{j}},\left.e_{i}\right|_{p_{j}}=0$ unless $j=i$ and

$$
\left.e_{i}\right|_{p_{i}}(t)=\prod_{j \neq i}\left(t^{a_{i}}-t^{a_{j}}\right)
$$

By Corollary 5.6, with $u=e_{i}$, we have

$$
\operatorname{Id}_{S^{1}}^{X}\left(\delta_{S^{1}} e_{i}\right)(t)=t^{-\lambda_{i}} \prod_{j \neq i}\left(t^{a_{i}}-t^{a_{j}}\right) \cdot \prod_{j=1}^{n}\left(1-t^{x_{i j}}\right)^{-1}
$$

and the result follows from (2.2).

LEMMA 2.3. $\psi_{k}(1)=\varepsilon$ where $\varepsilon= \pm 1$ is independent of $k$.

Proof. Since the normal bundle $v$ of $X^{S^{1}}$ in $X$ is trivially a complex bundle, there is a homomorphism $i_{*}: K_{S^{1}}^{*}\left(X^{S^{1}}\right) \rightarrow K_{S^{1}}^{*}(X)$ induced by the 
inclusion $i: X^{S^{1}} \rightarrow X$ and having the property that

$$
i^{*} i_{*}(x)=\lambda_{-1}(v) \cdot x \quad \text { for } x \in K_{S^{1}}^{*}\left(X^{S^{1}}\right) .
$$

See Part I, (1.1).

There is a commutative diagram (Part I, (1.3))

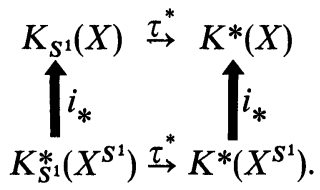

Since $X^{S^{1}}=\bigcup_{i=0}^{n} p_{j}$

$$
K_{S^{1}}\left(X^{S^{1}}\right)=\prod K_{S^{1}}^{*}\left(p_{j}\right) .
$$

Let $f_{k} \in K_{S^{1}}^{*}\left(p_{k}\right)$ be the identity and $x=\left(0,0, \ldots, f_{k}, \ldots, 0\right) \in K_{S^{1}}^{*}\left(X^{S^{1}}\right)$. Then $\tau^{*} f_{k}$ is the identity of $K^{*}\left(p_{k}\right)$ and $i_{*} \tau^{*}(x)=\varepsilon(\eta-1)^{n} ; \varepsilon$ depends on the orientation of $X$. This follows from the definition of $i_{*}$.

Let $\mathfrak{p}$ denote the prime ideal of characters which vanish at $t \in S^{1}$ and consider the element

$$
d_{k}=\prod_{j \neq k}\left(\eta-t^{a_{j}}\right)\left(t^{a_{k}}-t^{a_{j}}\right)^{-1} \prod_{j=1}^{n}\left(1-t^{x_{i j}}\right)
$$

of $\hat{K}_{S^{1}}(X)_{\mathfrak{p}}$. By the localization theorem $i_{\mathfrak{p}}^{*}: \hat{K}_{S^{1}}(X)_{\mathfrak{p}} \rightarrow K_{S^{1}}^{*}\left(X^{S^{1}}\right)_{\mathfrak{p}}$ is an isomorphism. But

$$
i_{\mathfrak{p}}^{*} d_{k}=\prod_{j=1}^{n}\left(1-t^{x_{i j}}\right) x=\lambda_{-1}(v) \cdot x
$$

and

$$
i_{\mathfrak{p}}^{*} i_{*}(x)=\lambda_{-1}(v) \cdot x .
$$

Since $i_{\mathfrak{p}}^{*}$ is a monomorphism, $i_{*}(x)=d_{k}$. Thus $d_{k} \in \hat{K}_{S^{1}}(X) \subset \hat{K}_{S^{1}}(X)_{p}$ so $\tau^{*} d_{k}$ is defined and

$$
\tau^{*}\left(d_{k}\right)=\psi_{k}(1)(\eta-1)^{n}
$$

and

$$
\psi_{k}(1)(\eta-1)^{n}=\tau^{*}\left(d_{k}\right)=\tau^{*} i_{*}(x)=i_{*} \tau_{*}(x)=\varepsilon(\eta-1)^{n}
$$

so $\psi_{k}(1)=\varepsilon$.

Corollary 2.4 .

$$
\prod_{j \neq i}\left(a_{j}-a_{i}\right)=\varepsilon \prod_{j=1} x_{i j}
$$


Proof.

$$
\begin{aligned}
\psi_{i}(1) & =\lim _{t \rightarrow 1} \prod_{j \neq i}\left(1-t^{a_{j}-a_{i}}\right) \prod_{j=1}^{n}\left(1-t^{x_{i j}}\right)^{-1} \\
& =\prod_{j \neq i}\left(a_{j}-a_{i}\right) / \prod_{j=1}^{n} x_{i j}=\varepsilon .
\end{aligned}
$$

DEFINITION 2.5. For each integer $m$ and each $i=0,1,2, \ldots, n$, set

$$
\begin{aligned}
& n_{i}(m)=\text { number of } j \neq i \text { such that } m \text { divides } a_{j}-a_{i}, \\
& d_{i}(m)=\text { number of } j=1,2, \ldots, n \text { such that } m \text { divides } x_{i j}, \\
& \delta_{i}(m)=n_{i}(m)-\delta_{i}(m) .
\end{aligned}
$$

THEOREM 2.6. $\delta_{i}(m) \geqq 0$ and $\delta_{i}\left(p^{r}\right)=0$ if $p$ is prime.

Proof. Let $\phi_{d}(t)=\prod_{\xi \in S_{d}}(t-\xi)$ where $S_{d}$ consists of the primitive $d$ th roots of unity, i.e., $\phi_{d}(t)$ is the $d$ th cyclotomic polynomial. Then

$$
\tau^{l}-1=\prod_{d \mid l} \phi_{d}(t)
$$

and

$$
\begin{aligned}
\psi_{i}(t) & = \pm t^{N} \prod_{m} \phi_{m}(t)^{n_{i}(m)} / \prod_{m} \phi_{m}(t)^{d_{i}(m)} \\
& = \pm t^{N} \prod_{m} \phi_{m}(t)^{\delta_{i}(m)}
\end{aligned}
$$

where $N$ is an integer. By Lemma 2.1, $\psi_{i}(t) \in \Lambda$. But each $\phi_{m}(t)$ defines a prime ideal in $\Lambda \otimes Q=Q\left[t, t^{-1}\right]$. Since $\psi_{i}(t) \in \Lambda \otimes Q, \delta_{i}(m) \geqq 0$. Next observe [7]

$$
\begin{aligned}
\phi_{m}(1) & =p, & & m=p^{r} p \text { prime } \\
& =1, & & m \text { composite }
\end{aligned}
$$

Thus

$$
\psi_{i}(1)= \pm \prod_{p, r} p^{\delta_{i}\left(p^{r}\right)}= \pm 1
$$

so $\delta_{i}\left(p^{r}\right)=0$.

REMARK. If $\delta_{i}(m)=0$ for all $m$ not just prime powers, then the two collections of integers

$$
\left\{\left|a_{j}-a_{i}\right| \mid j \neq i\right\}=\mathscr{S}_{i} \text { and } T_{i}=\left\{\left|x_{i j}\right| \mid j=1,2, \ldots, n\right\}
$$

are equal and this would establish the truth of

Statement 2.7. If $S^{1}$ acts smoothly on a manifold $X$ homotopy equivalent to $C P^{n}$ with $n+1$ isolated fixed points $p_{i}$, then the $n+1$ integers $a_{i}$ defined 
by $\left.\psi\right|_{p_{i}}(t)=t^{a_{i}}, t=e^{i \theta} \in S^{1}$ (Part II, Definition 1.2), and the integers $x_{i j}$, $j=1,2, \ldots, n$, defining the representation $\rho_{i}$ of $S^{1}$ on $\left.T X\right|_{p_{i}}$ by

$$
\rho_{i}\left(e^{i \theta}\right)=\operatorname{diag}\left(\begin{array}{rr}
\cos x_{i j} \theta & \sin x_{i j} \theta \\
-\sin x_{i j} \theta & \cos x_{i j} \theta
\end{array}\right), \quad j=1,2, \ldots, n,
$$

are related by $\left\{\left|x_{i j}\right| \mid j=1,2, \ldots, n\right\}=\left\{\left|a_{j}-a_{i}\right| \mid j \neq i\right\}$.

In fact, 2.7 is false, as we shall see in $\$ 4$; however, here are some additional relations which must hold between the $x_{i j}$ and the $\left(a_{j}-a_{i}\right)$.

THEOREM 2.8. The $n+1 \times n$ matrix of integers $\left(\left(x_{i j}\right)\right), i=0,1, \ldots, n$, $j=1,2, \ldots, n$, whose ith row gives the real representation of $S^{1}$ on $\left.T X\right|_{p}$; and the $n+1$ integers $\left\{a_{i}\right\}$ defined by $\left.\eta\right|_{p_{i}}(t)=t^{a_{i}}$ must satisfy these relations: There is an integer $N$ such that, for every integer $k$,

$$
\begin{gathered}
t^{N / 2} \cdot \sum_{i=0}^{n} t^{a_{i}(k+(n+1) / 2)} \cdot \prod_{j=1}^{n}\left(t^{-x_{i j} / 2}-t^{x_{i j} / 2}\right)^{-1} \in Z\left[t, t^{-1}\right] . \\
\prod_{j \neq i}\left(a_{j}-a_{i}\right)=\varepsilon \prod_{j=1} x_{i j} \quad \text { where } \varepsilon \text { is }+ \text { or }-1 \text { independent of } i . \\
\sum_{i=0}^{n} \prod_{j=1}^{n}\left(t^{x_{i j}}+1\right)\left(t^{x_{i j}}-1\right)^{-1}=0, \text { if } n \text { is odd, } \\
=1, \text { if } n \text { is even. }
\end{gathered}
$$

Proof. Let $L=(n+1) c_{1}\left(\eta^{\prime}\right)$ where again $\eta^{\prime}$ is the pull back of the Hopf bundle over $C P^{n}$. See Part II, Proposition 1.1. Since Stiefel-Whitney classes are preserved by homotopy equivalences, the $\bmod 2$ reduction of $L$ is the second Stiefel-Whitney class of $X$. By Part I, Corollary 3.8, there is a principle $\operatorname{spin}^{c}(2 n)$ bundle $P_{L}$ over $X$ with

$$
P_{L} \times{ }_{\operatorname{spin}^{c}(2 n)} C=\left(\eta^{\prime}\right)^{n+1} .
$$

Part (i) now follows from Part I, Theorem 6.5, by setting $W_{1}=\eta$ (Part II, Proposition 1.1), $z_{1}=c_{1}\left(\eta^{\prime}\right), z_{1 j}=a_{j}, L_{j}=(n+1) a_{j}$ and $\Phi\left(y_{1}\right)=y_{1}^{k}$. Part (ii) is a restatement of Corollary 2.4. Part (iii) is a consequence of the fact that the $S^{1}$ signature of $X[6, \mathrm{p} .578]$ as a function on $S^{1}$ is the constant 0 or 1 depending on $n$. The left-hand side of (iii) is the expression for the symbol of the index operator on $X$.

We return to the Remark following Part I, Theorem 6.5, as we want an explicit formula for $\operatorname{Id}_{S^{1}}^{X}\left(\delta_{S^{1}}, \Phi\right)(t)$ when $\Phi \in K_{S^{1}}^{*}(X)$ is a polynomial $\Phi(\eta)$ in $\eta$ involving only: the integers $a_{i}$, the integers $\left\{x_{j k}\right\}$ and the first Chern class of the line bundle $\omega=P \times_{\operatorname{spin}^{c}(2 n)} C$. That is, we want to remove the ambiguity in signs which occur in Part I, Theorem 6.5 (at least for the case at hand) due to the presence of square roots. To that end we introduce the expressions

$$
\xi_{i}(t)=\prod_{j \neq i}\left(t^{-\left(a_{j}-a_{i}\right) / 2}-t^{\left(a_{j}-a_{i}\right) / 2}\right) \prod\left(t^{-x_{i j} / 2}-t^{x_{i j} / 2}\right)^{-1} .
$$


Of course, $\xi_{i}(t)$ is only defined up to sign; however, we impose the additional restriction: There is an integer $M$ such that

$$
t^{M / 2} \cdot \sum_{i=0}^{n} \Phi_{i}(t) \xi_{i}(t) \prod_{j \neq i}\left(1-t^{a_{j}-a_{i}}\right)^{-1} \in Z\left[t, t^{-1}\right]
$$

for every $\Phi=\Phi(\eta) \in K_{S^{1}}^{*}(X)$. Here $\Phi_{i}(t)=\Phi\left(t^{a_{i}}\right)$.

LEMMA 2.9. The vector $v=\left(\xi_{0}(t), \xi_{1}(t), \ldots, \xi_{n}(t)\right)$ is well defined up to sign. That is, if the $\xi_{i}(t)$ satisfy $(*)$ and $\left({ }^{* *}\right)$ and if $\xi_{0}(t)$ is fixed, the ambiguity in sign in the remaining $\xi_{i}(t)$ disappears.

Proof. Let $v_{i}=\left(\xi_{0}^{i}(t), \xi_{1}^{i}(t), \ldots, \xi_{n}^{i}(t)\right), i=0,1$, be two distinct solutions of $(*)$ and $\left({ }^{* *}\right)$. Suppose $v_{0} \neq-v_{0}$. Then there must be a pair $k$ and $j$ with $\xi_{j}^{0}(t)=\xi_{j}^{1}(t)$ and $\xi_{k}^{1}(t)=-\xi_{k}^{0}(t)$. Let

$$
\Phi(\eta)=\prod_{i \neq k ; i \neq j}\left(\eta-t^{a_{i}}\right) \in K_{S^{1}}^{*}(X) .
$$

Then $\Phi_{i}(t)=0, i \neq k, j$. Apply $(* *)$ to $v_{0}$ and $v_{1}$ and add the two expressions obtaining the condition

$$
2 t^{M / 2} \cdot \Phi_{j}(t) \xi_{j}(t) \prod_{l \neq j}\left(1-t^{a_{l}-a_{j}}\right)^{-1} \in Z\left[t, t^{-1}\right] .
$$

But

$$
\Phi_{j}(t) \prod_{l \neq j}\left(1-t^{a_{l}-a_{j}}\right)^{-1}=t^{\alpha} /\left(1-t^{a_{k}-a_{j}}\right)
$$

and $\xi_{j}(t)=t^{\beta / 2} \cdot \psi_{j}(t)$ where $\alpha$ and $\beta$ are integers. By Lemma $2.3, \psi_{j}(t)$ has no zero at $t=1$. Thus

$$
\Phi_{j}(t) \xi_{j}(t) \cdot \prod_{l \neq j}\left(1-t^{a_{l}-a_{j}}\right)^{-1}
$$

has a pole at $t=1$ contradicting $(* * *)$.

The relationship between $(* *)$ and $\operatorname{Id}_{S^{1}}^{X}\left(\delta_{S^{1}}, \Phi\right)(t)$ is this

THEOREM 2.10. Let $P$ be the principle $\operatorname{spin}^{c}(2 n)$ bundle associated to $T X$ with $c_{1}(\omega)=(n+1) b$ where $\omega=P \times_{\operatorname{spin}^{c}(n)} C$ and $b=c_{1}\left(\eta^{\prime}\right)$. Let $\delta_{S^{1}}$ be the orientation class constructed from $P$. Then there is an integer $N$ such that, for every $\Phi=\Phi(\eta) \in K_{S^{1}}^{*}(X)$,

$$
\operatorname{Id}_{S^{1}}^{X}\left(\delta_{S^{1}} \Phi\right)(t)=t^{\left(N+\Sigma a_{k}\right) / 2} \sum_{i=0}^{n} \Phi_{i}(t) \xi_{i}(t) \prod_{j \neq i}\left(1-t^{a_{j}-a_{i}}\right)^{-1}
$$


Proof. By Part I, Theorem 6.5, with $L_{i}=(n+1) a_{i}$ and $\Phi_{i}(t)=\Phi\left(t^{a_{i}}\right)$ we have

$$
\begin{aligned}
E_{\Phi} & =\operatorname{Id}_{S^{1}}^{X}\left(\delta_{S^{1}} \Phi\right)(t) \\
& =t^{N / 2} \sum_{i=0}^{n} \Phi_{i}(t) t^{(n+1) a_{i} / 2} \prod_{j=1}^{n}\left(t^{-x_{i j} / 2}-t^{x_{i j} / 2}\right)^{-1} .
\end{aligned}
$$

The result follows by arithmetic.

THEOREM 2.11. Let $S^{1}$ act on a manifold homotopy equivalent to $C P^{n}$. If $X^{S^{1}}$ consists of isolated fixed points, the cohomology class $\hat{\mathscr{A}}(X) \in H^{*}(X, Q)$ is determined by the equivariant "Hopf bundle" $\eta$ and the integers $x_{j k}$ describing the representations of $S^{1}$ on $T X$ at the isolated fixed points.

Proof. Let

$$
E_{k}(t)=t^{\left(N+\Sigma a_{l}\right) / 2} \sum_{i=0}^{n} \frac{t^{a_{i} k} \xi_{i}(t)}{\prod_{j \neq i}\left(1-t^{a_{j}-a_{i}}\right)} .
$$

Since the $a_{i}$ are defined by $\left.\eta\right|_{p_{i}}(t)=t^{a_{i}}$, it follows from Lemma 2.10 that $E_{k}(t)$ is determined up to sign by $\eta$ and the $x_{j k}$ 's.

Let $b=c_{1}\left(\eta^{\prime}\right)$ be the first Chern class of $\eta^{\prime},[X] \in H_{2 n}(X)$ the orientation class and $\langle y,[X]\rangle$ the value of the cohomology class $y \in H^{*}(X, Q)$ evaluated on the orientation class. Then by the Remark following Part I, Proposition 5.3,

$$
\left\langle e^{(k+(n+1) / 2) b} \hat{A}(X),[X]\right\rangle=\operatorname{Id}_{S^{1}}^{X}\left(\eta^{k} \delta_{S^{1}}\right)(1)=\lim _{t \rightarrow 1} E_{k}(t)=E_{k}
$$

and $E_{k}$ is determined up to a sign independent of $k$ by the given data.

We observe that: $e^{((n+1) / 2) b}$ is a unit of $H^{*}(X, Q)$ because its degree zero term is $1, \operatorname{ch}: K^{*}(X) \otimes Q \rightarrow H^{*}(X, Q)$ is an isomorphism so $\left\{e^{k b}=c h \eta^{\prime k} \mid k\right.$ $=0,1, \ldots, n\}$ is a basis for $H^{*}(X, Q)$ and since $e^{((n+1) / 2) b}$ is a unit, $\left\{e^{(k+(n+1) / 2) b} \mid k=0,1, \ldots, n\right\}$ is also a basis and by Poincaré duality $\left\{e^{(k+(n+1) / 2) b} \cap[X]\right\}$ is a basis for $H_{*}(X, Q)$. Since the values of $\langle\mathscr{A}(X)$, $\left.e^{(k+(n+1) / 2) b} \cap[X]\right\rangle$ for $k=0,1,2, \ldots, n$ determine $\hat{A}(X), \hat{A}(X)$ is determined up to sign by the given data. However, $\hat{A}(X)=1+$ terms of higher dimension and this fixes $\mathscr{A}(X)$.

CoROllaRY 2.12. Suppose $\xi_{i}(t)=\xi(t)$ is independent of $i$. If $h: X \rightarrow C P^{n}$ is a homotopy equivalence, $h^{*} \mathscr{A}\left(C P^{n}\right)=\hat{\mathscr{A}}(X)$.

Proof. Let $x \in H^{2}\left(C P^{n}\right)$ be the first Chern class of the Hopf bundle and $b=h^{*}(x)$. Set

$$
D_{k}(t)=\sum_{i=0}^{n} t^{k a_{i}} \prod\left(1-t^{a_{j}-a_{t}}\right)^{-1}
$$


Then by Part I, (6.4) (iv) and (v) and the Remark following Part I, Proposition 2.8,

$$
\left\langle e^{((n+1) / 2+k) x} \hat{\mathscr{A}}\left(C P^{n}\right),\left[C P^{n}\right]\right\rangle=\operatorname{Id}_{S^{1}}^{C{ }^{n}}\left(\delta_{S^{1}} \mathscr{H}^{k}\right)(1)=D_{k}(1) .
$$

On the other hand, it follows from Theorem 2.10 that

$$
\left\langle e^{((n+1) / 2+k) b} \hat{\mathscr{A}}(X),[X]\right\rangle=\operatorname{Id}_{S^{1}}^{X}\left(\delta_{S^{1}} \eta^{k}\right)(1)=\xi(1) D_{k}(1) .
$$

Since $\xi_{i}(t)=t^{\gamma_{i} / 2} \cdot \psi_{i}(t)$ for some integer $\gamma_{i}$, it follows from Lemma 2.3 that $\xi(1)= \pm 1$. Thus

$$
\begin{aligned}
\left\langle h^{*} \mathscr{A}\left(C P^{n}\right),[X]\right. & \left.\cap e^{((n+1) / 2+k) b}\right\rangle \\
& = \pm\left\langle\hat{A}\left(C P^{n}\right),\left[C P^{n}\right] \cap e^{((n+1) / 2+k) b}\right\rangle \\
& = \pm\left\langle\hat{A}(X)[X] \cap e^{((n+1) / 2+k) b}\right\rangle .
\end{aligned}
$$

Since this holds for every integer $k, h^{*} \mathscr{A}\left(C P^{n}\right)= \pm \hat{\mathscr{A}}(X)$. However, $\hat{A}\left(C P^{n}\right)=1 \pm$ terms of higher dimension and likewise for $\mathscr{A}(X)$; so the plus sign must hold.

We end the section with an example due to G. Bredon which shows that the analogs of Lemma 1.9 and Corollary 1.14 are false without the assumption on the field $k$.

Let $S^{1}$ act on the complex plane $C^{2}$ via the representation $\rho=t^{2}+t^{3}$ $\in R\left(S^{1}\right)$. Then $S^{4}$ is the one point compactification of $C^{2}$ and the $S^{1}$ action extends uniquely to a smooth action on $S^{4}$ with fixed point set 0 and $\infty$. The isotropy subgroups are $0, Z_{2}, Z_{3}$ and $S^{1}$.

Let $\mathcal{O}$ be the $S^{1}$ orbit of the point $(1,1) \in C^{2}$ and $\mathfrak{T}$ be an open equivariant tubular neighborhood of $\mathcal{O}$. Set $X=S^{4}-\mathcal{O}$. Then $X$ is diffeomorphic to $S^{2} \times D^{2}=C P^{1} \times D^{2}$. Let $p_{1}=0$ and $p_{2}=\infty \in X$. The integers associated to $p_{1}$ and $p_{2}$ via $\left.\eta\right|_{p_{i}}(t)=t^{a_{i}}$ are $a_{1}$ and $a_{1}+6=a_{2}$. Thus $a_{2}-a_{1}=6$ but the fixed point set of $Z_{6}$ is the same as the fixed point set of $S^{1}$. Compare Corollary 1.14.

As an algebra

$$
K_{S^{1}}^{*}(X) \cong \Lambda[x] /\left(\left(x^{2}+\phi_{1} \phi_{2} \phi_{3} x\right)\right)
$$

where $\phi_{d}=\phi_{d}(t)$ is the $d$ th cyclotomic polynomial. The algebra $\Gamma$ in this case is

$$
\Gamma=\Lambda[y] /\left(\left(y^{2}-\left(1-t^{6}\right) y\right)=\Lambda[\bar{y}] /\left((\bar{y}-1)\left(\bar{y}-t^{6}\right)\right)\right.
$$

where $\bar{y}=y+t^{6}$.

Let $k$ be the cyclotomic field of primitive sixth roots of unity. The map of algebras $A$ from $\Gamma$ to $K_{S^{1}}^{*}(X)$ is defined by $A(y)=\phi_{6}(t) x$, so $A \otimes_{\Lambda} 1_{k}$ : $\Gamma \otimes_{\Lambda} k \rightarrow K_{S^{1}}^{*}(X) \otimes_{\Lambda} k$ is not an isomorphism. Compare Lemma 1.9.

3. Speculation: Bilinear forms on $K_{G}^{*}(X)$. When $X$ is a closed oriented manifold of dimension $2 n$, the cup product pairing on $\hat{H}^{n}(X, Z)$ 
$=H^{n}(X, Z) /$ Torsion is nondegenerate. This means that the homomorphism $\Phi: \hat{H}^{n}(X, Z) \rightarrow \operatorname{Hom}_{Z}\left(\hat{H}^{n}(X, Z), Z\right)$ defined by $\Phi(x)[y]=\langle x \cup y$, $[X]>$ for $x, y \in \hat{H}^{n}(X, Z)$ and $[X] \in H_{2 n}(X, Z)$ the orientation class is an isomorphism. This is a consequence of

(a) Poincaré duality $\hat{H}^{n}(X, Z) \cong \hat{H}_{n}(X, Z)$.

(b) The universal coefficient theorem: Cap product defines an isomorphism; $\hat{H}^{n}(X, Z) \rightarrow \operatorname{Hom}_{Z}\left(\hat{H}_{n}(X), Z\right)$.

The statement of the universal coefficient theorem may be turned around to

(b) $\hat{H}_{n}(X, Z) \rightarrow \operatorname{Hom}_{Z}\left(\hat{H}^{n}(X, Z), Z\right)$ is an isomorphism.

The concept of the nondegenerate bilinear form constructed in this way has been a powerful tool in the development of the topology of manifolds. The purpose of this section is to set up an analogous situation for $K_{G}^{*}(X)$, ask some questions and discuss examples.

Suppose that $G$ is a compact connected Lie group acting smoothly on a closed $G$ oriented manifold $X$. Then $K_{*}^{G}(X)=K_{G}^{*}(T X)$ and Poincaré duality holds; i.e., we have an isomorphism $\psi_{G}: K_{G}^{*}(X) \rightarrow K_{G}^{*}(T X)$. In addition we have an $R(G)$ homomorphism

$$
\hat{D}: K_{G}^{*}(T X) \rightarrow \operatorname{Hom}_{R(G)}\left(K_{G}^{*}(X), R(G)\right)
$$

defined by

$$
\hat{D}(x)[y]=\operatorname{Id}_{G}^{X}(x \cdot y), \quad x \in K_{G}^{*}(T X), \quad y \in K_{G}^{*}(X) .
$$

The composition $\hat{D} \psi_{G}$ gives rise to a bilinear form $\left\langle>\right.$ on $\hat{K}_{G}(X)=K_{G}^{*}(X) / \mathfrak{I}$ where $\mathfrak{I}$ is the $R(G)$ torsion subgroup of $K_{G}^{*}(X)$. Precisely

$$
\langle z, y\rangle=\operatorname{Id}_{G}^{X}\left(\psi_{G}(z) \cdot y\right)=\hat{D}\left(\psi_{G}(z)\right)[y]
$$

for $z, y \in K_{G}^{*}(X)$.

Question 3.2. When is the bilinear form $\left\langle>\right.$ on $\hat{K}_{G}(X)$ nondegenerate? This means that the map $\hat{K}_{G}(X) \rightarrow \operatorname{Hom}_{R(G)}\left(\hat{K}_{G}(X), R(G)\right)$ defined by $\hat{D} \psi_{G}$ is an isomorphism of $R(G)$ modules.

Question 3.3. When the preceding question has an affirmative answer, can one relate the algebraic invariants of the bilinear form \langle\rangle to the representations of $G$ on the fibers normal to the fixed point sets?

One hopes that the bilinear form $\left\langle>\right.$ is nondegenerate when $\hat{K}_{G}(X)$ is free over $R(G)$. We discuss some interesting cases when this is true.

Case 3.4. $X=U(Z)$ is the group of isometries of a complex $G$ module $Z$ and $G$ acts on $X$ by

$$
g \cdot x=\rho(g) x \rho(g)^{-1}, \quad g \in G, x \in U(Z),
$$

where $\rho: G \rightarrow U(Z)$ is the representation given by the structure of $Z$ as a complex $G$ module. In Part I, $\$ 4$, Example 3, we constructed an orientation class $\alpha_{G}$. See Part I, Proposition 4.11. 
Proposition 3.5. The bilinear form $\left\langle>\right.$ on $\hat{K}_{G}(X)$ defined by

$$
\langle x, y\rangle=\operatorname{Id}_{G}^{X}\left(\alpha_{G} x \cdot y\right)
$$

is nondegenerate.

Proof. Suppose the complex dimension of $Z$ is $n$. Then

$$
K_{G}^{*}(X)=R(G) \otimes_{Z} \Lambda\left(\theta_{1}, \ldots, \theta_{n}\right)
$$

is the exterior algebra over $R(G)$ generated by $n$ basic generators $\theta_{1}, \theta_{2}, \ldots, \theta_{n}[\mathbf{1 0}]$. Here $\Lambda\left(\theta_{1}, \ldots, \theta_{n}\right)$ is the $Z$ exterior algebra generated by $\theta_{1}, \theta_{2}, \ldots, \theta_{n}$. Moreover, the homomorphism $\rho^{*}: K_{U}^{*}(Z)(X) \rightarrow K_{G}^{*}(X)$ induced by the homomorphism $\rho: G \rightarrow U(Z)$ is given by $\rho^{*}=\tilde{\rho} \otimes 1$ where $\tilde{\rho}: R U(Z) \rightarrow R(G)$ is induced by the homomorphism $\rho$. This means that a $Z$ basis $\left\{e_{i}\right\}$ for $\Lambda\left(\theta_{1}, \ldots, \theta_{n}\right)$ gives an $R(G)$ basis for $K_{G}^{*}(X)$ and $K_{U(Z)}^{*}(X)$. Note that \langle\rangle is nondegenerate on $K_{G}^{*}(X)$ if and only if the determinant of the matrix $\left\langle e_{i}, e_{j}\right\rangle$ is a unit of $R(G)$. Let us denote this determinant by $\operatorname{det}_{R(G)}\left\langle e_{i}, e_{j}\right\rangle$. By the above remarks and the compatibility axiom (Part I, (1.3))

$$
\begin{aligned}
\operatorname{det}_{R(G)}\left\langle e_{i}, e_{j}\right\rangle & =\operatorname{Id}_{G}^{X}\left(\alpha_{G} e_{i} \cdot e_{j}\right) \\
& =\operatorname{Id}_{G}^{X}\left(\rho^{*} \alpha_{U(Z)} e_{i} \cdot e_{j}\right)=\tilde{\rho} \operatorname{Id}_{U(Z)}^{X}\left(\alpha_{U(Z)} e_{i} \cdot e_{j}\right) \\
& =\tilde{\rho} \operatorname{det}_{R(U(Z))}\left\langle e_{i}, e_{j}\right\rangle .
\end{aligned}
$$

Thus it suffices to prove the proposition when $G=U(Z)$. For convenience we set $U(Z)=U$. As properties of the maximal torus of $U$ play a key role in our proof we discuss them before proceeding.

Let $j: \mathfrak{T} \rightarrow U$ be the inclusion of the maximal torus $\mathfrak{I}$ viewed as a homomorphism of groups and $i: \mathfrak{T} \rightarrow U=X$ the same map viewed as a continuous map of topological spaces. Let $\mathfrak{I}$ operate on itself and $X$ via inner automorphisms i.e., $x \in X, t \in \mathfrak{T}$,

$$
t \circ x=t x t^{-1} \text {. }
$$

Then $i$ is equivariant with respect to the action of $\mathfrak{I}$ and $X^{\mathfrak{T}}=\mathfrak{I}$ i.e., the fixed point set of the action of $\mathfrak{I}$ on $X$ is $i \mathfrak{I}$ which we briefly write as $\mathfrak{I}$.

Since $\mathfrak{I}$ acts trivially on itself, $T \mathfrak{I}=\mathfrak{I} \times W$ where $W=R^{n}$ with trivial action of $\mathfrak{T}$. Either $W$ or $W \times R^{1}$ is a complex $\mathfrak{T}$ module $\hat{W} \cong C^{[(n+1) / 2]}$ with trivial action and the class

$$
\begin{aligned}
\alpha_{\mathfrak{I}} & =1 \otimes \Delta_{\mathfrak{T}} \in K_{\mathfrak{I}}^{0}(\mathfrak{I}) \otimes_{R(\mathfrak{I})} K_{\mathfrak{T}}^{0}(\hat{W}) \\
& =K_{\mathfrak{T}}^{0}(\mathfrak{I} \times \hat{W})
\end{aligned}
$$

is an orientation for $\mathfrak{T}$. See Part I, $\S 4$, Example 3. Moreover, if $\hat{V}$ is the complex $U$ module used in constructing $\Delta_{U}$ we have

$$
\left.\hat{V}\right|_{\mathfrak{I}}=\hat{W} \oplus O
$$


where $O$ is the tangent space of $U / \mathfrak{I}$ at $[\mathfrak{T}] \in U / \mathfrak{I}$. The representation of $\mathfrak{I}$ on $O$ is given by the adjoint action. This gives a representation $\omega: \mathfrak{T}$ $\rightarrow U(d)$ where $2 d=$ real $\operatorname{dim} O$ and the composition of $\omega$ with the map $\psi_{0}: U(d) \rightarrow \operatorname{spin}^{c}(2 n)\left(\right.$ Part I, (2.3)) gives a homomorphism $\widetilde{A d}=\psi_{0} \omega: \mathfrak{I}$ $\rightarrow \operatorname{spin}^{c}(2 n)$. Let $\Omega=A d^{*}\left(\Delta_{+}-\Delta_{-}\right) \in R(\mathfrak{I})$.

It follows from (3.6) that

$$
s^{*} j^{*} \Delta_{U}=\Delta_{\mathfrak{I}} \cdot \Omega \in K_{\mathfrak{I}}^{0}(\hat{W})=K_{\mathfrak{I}}^{[(n+1) / 2]}(T \mathfrak{T}) .
$$

Here $s: \hat{W} \rightarrow \hat{W} \oplus O$ is the zero section of this bundle over $\hat{W}$.

Let $T i: T \mathfrak{I} \rightarrow T U$ be the inclusion which is equivariant with respect to the $\mathfrak{T}$ action on each. Then from the factorization

$$
K_{\mathfrak{x}}^{*}(T U)=K_{\mathfrak{I}}^{*}(U \times \hat{V}) \stackrel{i *}{\rightarrow} K_{\mathfrak{I}}^{*}(\mathfrak{T} \times(\hat{W} \oplus O)) \stackrel{s^{*}}{\rightarrow} K_{\mathfrak{x}}^{*}(\mathfrak{T} \times \hat{W})=K_{\mathfrak{x}}^{*}(T \mathfrak{T})
$$

of $T i^{*}$ and from (3.7) we see that

$$
T i^{*} j^{*} \alpha_{U}=\Omega \cdot \alpha_{\mathfrak{x}}
$$

Let $U$ act trivially on $\mathfrak{I}$ and by left translation on $U / \mathfrak{I}$. Then we have an equivariant map

$$
\pi: U / \mathfrak{I} \times \mathfrak{I} \rightarrow U
$$

defined by

$$
\pi(u \mathfrak{T}, t)=u t u^{-1}
$$

for $t \in \mathfrak{I}$ and $u \in U$. Note that $\mathfrak{I}$ is included in $U / \mathfrak{I} \times \mathfrak{I}$ via $f(t)=[\mathfrak{I}] \times t$. This is equivariant with respect to the $\mathfrak{I}$ action. We then have a commutative diagram of $\mathfrak{I}$ spaces and $\pi$ is $U$ equivariant

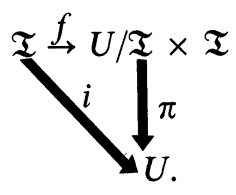

Atiyah [0] has shown that the coordinates of $\mathfrak{I}$ define elements $\beta_{i} \in K^{1}(\mathfrak{I})$ such that

(i) $\pi^{*}\left(\theta_{1} \cdot \theta_{2} \cdots \theta_{n}\right)=\xi^{\prime} \Omega \otimes \beta_{1} \cdot \beta_{2} \cdots \beta_{n} \in R(\mathfrak{I}) \otimes_{\mathbb{Z}} K^{*}(\mathfrak{I})=$ $K_{U}^{*}(U / \mathfrak{I} \times \mathfrak{T})$ where $\xi^{\prime}$ is a unit of $R(\mathfrak{I})$.

Let $\phi: \mathfrak{I} \rightarrow 1$ be the trivial homomorphism. Then because $\mathfrak{I}$ acts on itself trivially,

(ii) $\operatorname{Id}_{\mathfrak{T}}^{\mathfrak{T}}\left(\alpha_{\mathfrak{T}} \beta_{1} \cdots \beta_{n}\right)=\operatorname{Id}_{\mathfrak{T}}^{\mathfrak{T}}\left(\phi^{*} \alpha_{1} \beta_{1} \cdots \beta_{n}\right)=\phi \operatorname{Id}_{1}^{\mathfrak{T}}\left(\alpha_{1} \beta_{1} \cdots \beta_{n}\right)=1$.

By the localization theorem (Part I, 1.6), 
because

$$
\begin{aligned}
& \operatorname{Id}_{\mathfrak{I}}^{X}\left(j^{*} \alpha_{U} \theta_{1} \cdot \theta_{2} \cdots \theta_{n}\right) \\
= & \operatorname{Id}_{\mathfrak{x}}^{\mathfrak{T}}\left(T i^{*} j^{*} \alpha_{U} \theta_{1} \cdots \theta_{n} / \lambda_{-1}(0 \otimes C)\right) \\
= & \operatorname{Id}_{\mathfrak{x}}^{\mathfrak{T}}\left(\Omega \alpha_{\mathfrak{I}} \cdot \xi^{\prime} \Omega \beta_{1} \cdots \beta_{n} / \lambda_{-1}(0 \otimes C)\right)
\end{aligned}
$$

$$
\begin{aligned}
T i^{*} j^{*} \alpha_{U} \theta_{1} \cdots \theta_{n} & =\left(T i^{*} j^{*} \alpha_{U}\right) \cdot\left(i^{*} \theta_{1} \cdots \theta_{n}\right) \\
& =\Omega \alpha_{\mathfrak{x}} \cdot f^{*} \pi^{*} \theta_{1} \cdots \theta_{n}=\xi^{\prime} \Omega^{2} \cdot \alpha_{\mathfrak{x}} \cdot \beta_{1} \cdots \beta_{n}
\end{aligned}
$$

by (3.8) and (i). Let - be the involution of $R(\mathfrak{I})$ defined by sending a complex $\mathfrak{I}$ module $W$ to $\operatorname{Hom}_{\boldsymbol{C}}(W, C)$. Then a basic property of the element $\Omega$ is that $\Omega \bar{\Omega}=\lambda_{-1}(0 \otimes C)$ and $\bar{\Omega}=\xi \cdot \Omega$ where $\xi$ is a unit of $R(\mathfrak{I})[8]$. Putting all this information together gives

(iii) $\operatorname{Id}_{\mathfrak{z}}^{X}\left(j^{*} \alpha_{U} \theta_{1} \cdots \theta_{n}\right)=\xi^{\prime} \xi^{-1}$ a unit of $R(\mathfrak{I})$.

Note that $\tilde{j}$ is an inclusion of $R(U)$ in $R(\mathfrak{I})$ as the subring of invariants of the Weyl group. This implies that if $x \in R(U)$ and $\tilde{j}(x)$ is a unit of $R(\mathfrak{I})$, then $x$ is a unit of $R(U)$. From this and

(iv) $\tilde{j}^{\prime} \operatorname{Id}_{U}^{X}\left(\alpha_{U} \theta_{1} \cdots \theta_{n}\right)=\xi^{\prime} \xi^{-1}$ by (iii,) we see that

(v) $\operatorname{Id}_{U}^{X}\left(\alpha_{U} \theta_{1} \ldots \theta_{n}\right)$ is a unit of $R(U)$.

It follows from (v) and the algebra structure of the exterior algebra $\Lambda\left(\theta_{1}, \ldots, \theta_{n}\right)$ that the map $\hat{D} \psi_{U}: K_{U}^{*}(X) \rightarrow \operatorname{Hom}_{R(U)}\left(K_{U}^{*}(X), R(U)\right)$ is an isomorphism and this completes the proof of Proposition 3.5. Compare [0].

Proposition 3.9. Let $S^{1}$ act on $C P^{n}$ as described in Part I, (6.4) (Standard example). Then the bilinear form $\langle>$ is nondegenerate.

Proof. Atiyah has shown [1]

$$
K_{S^{1}}^{*}\left(C P^{n}\right)=R\left(S^{1}\right)[\mathscr{H}] /\left(\prod_{i=0}^{n}\left(\mathscr{H}-t^{a_{i}}\right)\right)
$$

as an $R\left(S^{1}\right)$ algebra. The restriction homomorphism

is given by

$$
i^{*}: K_{S^{1}}^{*}\left(C P^{n}\right) \rightarrow \prod_{i=0}^{n+1} K_{S^{1}}^{*}\left(p_{i}\right)=\prod_{i=0}^{n+1} R\left(S^{1}\right)
$$

$$
i^{*} \mathscr{H}^{k}=\left(t^{k a_{0}}, t^{k a_{1}}, \ldots, t^{k a_{n}}\right) \text {. }
$$

Thus $1, \mathscr{H}, \ldots, \mathscr{H}^{n}$ is a basis for $K_{S^{1}}^{*}\left(C P^{n}\right)$ over $R\left(S^{1}\right)$ and $i^{*}$ is a monomorphism and induces an isomorphism

$$
K_{S^{1}}^{*}\left(C P^{n}\right) \otimes_{R\left(S^{1}\right)} F\left(S^{1}\right) \rightarrow \prod_{i=0}^{n} F\left(S^{1}\right)
$$

where $F\left(S^{1}\right)$ is the field of fractions of $R\left(S^{1}\right)$. Let

$$
e_{i}=\prod_{j \neq i}\left(t^{a_{i}}-t^{a_{j}}\right)^{-1}\left(\mathscr{H}-t^{a_{j}}\right) .
$$


Then $e_{0}, e_{1}, \ldots, e_{n}$ is a base for $K_{S^{1}}^{*}\left(C P^{n}\right) \otimes_{R\left(S^{1}\right)} F\left(S^{1}\right)$ and

$$
\mathscr{H}^{k}=\sum t^{k a_{i}} e_{i}
$$

because of (3.10) and the fact that $i^{*}$ induces an isomorphism over $F\left(s^{\prime}\right)$.

Observe that $e_{i}^{2}=e_{i}$ because $i^{*} e_{i}^{2}=i^{*} e_{i} \cdot i^{*} e_{i}=i^{*} e_{i}$. Thus in terms of this new basis $\langle>$ gives a diagonal matrix with

$$
\left\langle e_{i}, e_{i}\right\rangle=\prod_{j \neq i}\left(1-t^{a_{j}-a_{i}}\right)^{-1} .
$$

This follows from Part I, Corollary 5.6, by setting $u_{j}(t)=\left.e_{i}\right|_{p_{j}}(t)$. Then $u_{i}(t)=1$ and $u_{j}(t)=0$ for $j \neq i$. Furthermore, $\lambda_{j}=0$ because the action of $S^{1}$ on the principle $\operatorname{spin}^{c}(2 n)$ bundle $P$ is defined using the lifting $\psi_{0}$ of $U(n)$ to $\operatorname{spin}^{c}(2 n)$ (Part I, 6.3).

Thus if $\Delta_{i}=\prod_{j \neq i}\left(t^{a_{i}}-t^{a_{j}}\right)$, then

$$
\operatorname{det}\left(\left(\left\langle e_{i}, e_{j}\right\rangle\right)\right)=t^{n \Sigma a_{j}} \cdot \prod_{i=0}^{n} \Delta_{i}^{-1} .
$$

Let $e_{i}=\sum_{j} S_{i j} \mathscr{H}^{j}$. Then

$$
\operatorname{det}\left(\left(\left\langle\mathscr{H}^{i}, \mathscr{H}^{j}\right\rangle\right)\right)=\operatorname{det} S^{-2} \operatorname{det}\left(\left(\left\langle e_{i}, e_{j}\right\rangle\right)\right) .
$$

By (3.11), $S$ is the inverse of the Vandermone matrix $V$ so $S^{-1}$ is $V$ and

$$
\operatorname{det} S^{-1}=\operatorname{det} V=\prod_{j<k}\left(t^{a_{j}}-t^{a_{k}}\right) .
$$

But

$$
(\operatorname{det} V)^{2}=\prod_{k} \prod_{j \neq k}\left(t^{a_{j}}-t^{a_{k}}\right)=\prod_{k=0}^{n} \Delta_{k} .
$$

Hence $\operatorname{det}\left(\left(\left\langle\mathscr{H}^{i}, \mathscr{H}^{j}\right\rangle\right)\right)=t^{n \Sigma a_{j}}$, a unit of $R\left(S^{1}\right)$. Since $\mathscr{H}^{i}, i=0,1,2, \ldots, n$, is a free $R\left(S^{1}\right)$ base for $K_{S^{1}}^{*}\left(C P^{n}\right)$, the proof is complete.

Finally, I mention the interesting analysis of Vasquez [18]. He studies the case in which $H \subset G$ are compact connected Lie groups with $H$ of maximal rank in $G$ and such that the homogenous space $X=G / H$ has a $\operatorname{spin}^{c}(m)$ structure, $m=\operatorname{dim} X$. Left translation by $G$ makes $X$ a $G$ space and Vasquez shows that the bilinear form \langle\rangle is nondegenerate on $K_{G}^{*}(X)$.

These examples can be multiplied by taking cartesian products. Anyway, Question 3.2 has an affirmative answer in enough cases to make it interesting and hopefully useful.

4. An exotic action of $S^{1}$ on $C P^{3}$. We offer an example of an action of $S^{1}$ on $C P^{3}$ which is definitely distinct from the linear actions of Part I, (6.4). This example is distinguished from the linear actions by the representation of $S^{1}$ on the tangent space at the four isolated fixed points. 
Representations of $S^{1}$ in $U(2)$ are defined by the

$$
\begin{array}{ll}
\omega(t)=\left(\begin{array}{ll}
t & 0 \\
0 & t^{6}
\end{array}\right), & \psi(t)=\left(\begin{array}{ll}
1 & 0 \\
0 & t^{7}
\end{array}\right), \\
\beta(t)=\left(\begin{array}{cc}
t^{2} & 0 \\
0 & t^{3}
\end{array}\right), & t \in S^{1} .
\end{array}
$$

The sphere $S^{3}$ is identified with $S U(2)$ via the map

$$
f(\vec{z})=\left(\begin{array}{rr}
z_{0} & z_{1} \\
-\bar{z}_{1} & \bar{z}_{0}
\end{array}\right), \quad \vec{z}=\left(z_{0}, z_{1}\right), \quad z_{i} \in C .
$$

We let $\vec{z} \cdot u$ for $\vec{z} \in S^{3}$ and $u \in U(2)$ denote the standard action of $U(2)$ on $S^{3}$.

LEMMA 4.1. In $U(2)$ we have

$$
\psi(t) f(\vec{z} \cdot \omega(t))=f(\vec{z}) \omega(t) .
$$

LEMMA 4.2. There is a degree one map $\Phi: S^{3} \rightarrow S^{3}$ satisfying

$$
\Phi(\vec{z} \cdot \beta(t))=\Phi(\vec{z}) \omega(t) .
$$

ProOF. Let

$$
\Phi\left(z_{0}, z_{1}\right)=\frac{\left(\bar{z}_{0} z_{1}, z_{0}^{3}+z_{1}^{2}\right)}{\left\|\left(\bar{z}_{0} z_{1}, z_{0}^{3}+z_{1}^{2}\right)\right\|}
$$

for $\left(z_{0}, z_{1}\right) \in S^{3}$. Here $\bar{z}_{0}$ denotes the complex conjugate of $z_{0}$.

Define a diffeomorphism $g: U(2) \times S^{3} \rightarrow U(2) \times S^{3}$ by

$$
g(u, \vec{z})=(u f(\Phi(\vec{z})), \vec{z}) .
$$

Let $X_{i}, i=0,1$, be the $S^{1}$ manifold whose underlying space is $U(2) \times D^{4}$ with $S^{1}$ action defined by

$$
\begin{array}{ll}
(u, \vec{z}) t=(u \psi(t), \vec{z} \beta(t)), & i=0, \\
(u, \vec{z}) t=(u \omega(t), \vec{z} \beta(t)), & i=1,
\end{array}
$$

$u \in U(2),|\vec{z}| \leqq 1$.

LEMMA 4.3. $g: \partial X_{0} \rightarrow \partial X_{1}$ is an equivariant diffeomorphism.

Proof. Equivariance follows from Lemmas 4.1 and 4.2. Since $g$ is 1-1, it is a diffeomorphism.

Let $H=U(1) \times U(1) \subset U(2)$. Then $H$ acts on the left of $U(2)$ by left multiplication and on $X_{i}$ by $\alpha(u, \vec{z})=(\alpha u, \vec{z}), \alpha \in U(1) \times U(1), u \in U(2)$ and $\vec{z} \in D^{4}$. The left action of $H$ on $X_{i}$ commutes with the right action of $S^{1}$ on $X_{i}$ so the orbit space $\bar{X}_{i}=X_{i} / H$ inherits an $S^{1}$ action. Moreover $g$ commutes with the action of $H$ on $\partial X_{0}$ and $\partial X_{1}$ and induces a diffeomorphism 
$\bar{g}: \partial \bar{X}_{0} \rightarrow \partial \bar{X}_{1}$ which is equivariant with respect to the $S^{1}$ action on each.

In a similar manner the left action of $H$ on $U(2)$ commutes with the two right actions of $S^{1}$ defined by

$$
u \circ t=u \psi(t), \quad u \circ t=u \omega(t),
$$

for $u \in U(2), t \in S^{1}$. The orbit space $U(2) / H$ is $C P^{1}=S^{2}$ and inherits two $S^{1}$ actions. We let $Z_{i}$, for $i=0,1$, denote the resulting $S^{1}$ manifolds.

Observe that as an $S^{1}$ manifold $\bar{X}_{i}=Z_{i} \times D(M)$ where $D(M)$ is the unit disk in the complex 2 dimensional $S^{1}$ module $M$ with $S^{1}$ acting via the representation $t \rightarrow \beta(t) \in U(2)$. This means that $\bar{X}_{i}$ is the total space of a disk bundle of a complex $S^{1}$ bundle over $Z_{i}$. Thus we have a Thom isomorphism

$$
\lambda_{i}: K_{S^{1}}^{*}\left(Z_{i}\right) \rightarrow K_{S^{1}}^{*}\left(\bar{X}_{i}, \partial \bar{X}_{i}\right) .
$$

LemMa 4.4. Let $X=\bar{X}_{0} \cup_{\bar{g}} \bar{X}_{1}$ denote the manifold obtained by identifying $x \in \partial \bar{X}_{0}$ with $\bar{g}(x) \in \partial \bar{X}_{1}$. Then $X$ is homotopy equivalent to $C P^{3}$.

PRoof. Since $\Phi: S^{3} \rightarrow S^{3}$ is homotopic to the identity (Lemma 4.2), $\bar{g}$ is homotopic to the map $H: S^{2} \times S^{3} \rightarrow S^{2} \times S^{3}$ defined by $H(u, \vec{z})=(u f(\vec{z})$, $z$ ) and $C P^{3}=S^{2} \times D^{4} \cup_{H} S^{2} \times D^{4}$.

The manifold $X$ inherits a unique $S^{1}$ action compatible with the given action on $\bar{X}_{i}$, for $i=0,1$. The fixed point set of this action consists of four isolated points $p_{0}, p_{1}, p_{2}, p_{3}$ labeled so that $p_{0}, p_{1} \in \bar{X}_{0}$ and $p_{2}, p_{3} \in \bar{X}_{1}$. The representations of $S^{1}$ on $\left.T X\right|_{p_{i}}$ are given by

$$
\begin{aligned}
& \left.T X\right|_{p_{0}}(t)=t^{7} \oplus t^{2} \oplus t^{3}, \\
& \left.T X\right|_{p_{1}}(t)=t^{-7} \oplus t^{2} \oplus t^{3}, \\
& \left.T X\right|_{p_{2}}(t)=t^{5} \oplus t^{2} \oplus t^{3}, \\
& \left.T X\right|_{p_{3}}(t)=t^{-5} \oplus t^{2} \oplus t^{3} .
\end{aligned}
$$

Actually we have listed complex representations which define the real representations we seek.

Here is an explicit description of the $S^{1}$ bundle $\eta$ over $X$. The total space of $\eta, E(\eta)$ is given as

$$
E(\eta)=C^{1} \times_{H} X_{0} \cup_{G} C^{1} \times_{H} X_{1}
$$

where $\left[c, x_{0}\right]$ in $C^{1} \times_{H} \partial X_{0}$ is identified with $G\left[c, x_{0}\right]=\left[c, g\left(x_{0}\right)\right]$ in $C^{1} \times_{H} \partial X_{1}$. The action of $E(\eta)$ is defined by the condition

$$
\left[c, x_{i}\right] t=\left[c, x_{i} t\right], \quad x_{i} \in X_{i}, i=0,1 .
$$

$c \in C^{1}, t \in S^{1}$. The projection $\pi$ of $E(\eta)$ on $X$ is described by $\pi\left[c, x_{i}\right]=p_{i}\left(x_{i}\right)$, $i=0,1$, and $p_{i}: X_{i} \rightarrow \bar{X}_{i}$ is the orbit map. We find 


$$
\left.\eta\right|_{p_{0}}(t)=t^{0},\left.\quad \eta\right|_{p_{1}}(t)=t^{7},\left.\quad \eta\right|_{p_{2}}(t)=t^{1},\left.\quad \eta\right|_{p_{3}}(t)=t^{6}
$$

Corollary 4.5. This action of $S^{1}$ on $X$ is not equivalent to any linear action of $S^{1}$ on $C P^{3}$. It is distinguished from such a linear action by the representations of $S^{1}$ on $T X$ at the isolated fixed points $p_{i}$.

Proof. By Part I, (6.4), the representations of $S^{1}$ on $\left.T C P\right|_{p_{i}}$ are completely determined by the equivariant Hopf bundle $\mathscr{H}$ as follows: $\left.\mathscr{H}\right|_{p_{i}}(t)=t^{a_{i}}$ and $\left.T X\right|_{p_{i}}(t)$ is the real representation defined by the complex representation $\chi_{i}=\sum_{j \neq i} t^{a_{j}-a_{i}}$.

Suppose $F: X \rightarrow C P^{3}$ is a diffeomorphism equivariant with respect to some linear action on $C P^{3}$. By composing $F$ with the map which conjugates the coordinates in $C P^{3}$ if necessary, we may suppose that $F$ is orientation preserving. Then by Stewart's theorem

$F^{*} \mathscr{H}=t^{k} \cdot \eta$ as $S^{1}$ line bundles for some integer $k$. Then $a_{0}=k$, $a_{1}=7+k, a_{2}=1+k, a_{3}=6+k$. But the real representations on $T X_{p_{i}}$ are not of the form

$$
\rho_{i}\left(e^{i \theta}\right)=\operatorname{diag}\left(\begin{array}{rr}
\cos \left(a_{k}-a_{i}\right) \theta & \sin \left(a_{k}-a_{i}\right) \theta \\
-\sin \left(a_{k}-a_{i}\right) \theta & \cos \left(a_{k}-a_{i}\right) \theta
\end{array}\right), \quad k \neq 1,
$$

as dictated by the linear case.

Corollary 4.6. $X$ is diffeomorphic to $C P^{3}$.

ProOF.

$$
\xi_{i}(t)=\frac{\left(t^{-1 / 2}-t^{1 / 2}\right)\left(t^{-6 / 2}-t^{6 / 2}\right)}{\left(t^{-2 / 2}-t^{2 / 2}\right)\left(t^{-3 / 2}-t^{3 / 2}\right)}=\xi(t)
$$

is independent of $i$. Choose an orientation preserving homotopy equivalence $h: X \rightarrow C P^{3}$. By Corollary 2.12, $h^{*} \hat{\mathscr{A}}\left(C P^{3}\right)=\hat{\mathscr{A}}(X)$ and $\hat{\mathscr{A}}(X)$ $=1-p_{1} h^{*}\left(x^{2}\right) / 24$ where $x$ is the first Chern class of the Hopf bundle and $p_{1}$ is an integer such that $p_{1} h^{*}\left(x^{2}\right)$ is the first Pontrjagin class of $X, P_{1}(X)$. Montgomery-Yang have shown [19] that the manifolds homotopy equivalent to $C P^{3}$ are in 1-1 correspondence with the integers. The correspondence is characterized by $i \rightarrow W_{i}$ where $P_{1}\left(W_{i}\right)=(24 i+4) h_{i}^{*}\left(x^{2}\right)$ and $h_{i}: W_{i} \rightarrow C P^{3}$ is a homotopy equivalence.

Since $h^{*} \hat{A}\left(C P^{3}\right)=\hat{\mathscr{A}}(X)$ we have $P_{1}(X)=4 h^{*}\left(x^{2}\right)$ so by the Montgomery-Yang theorem $X$ is diffeomorphic to $C P^{3}$.

5. The bilinear form $\left\langle>\right.$ on $K_{S^{1}}^{*}(X), X=C P^{3}$. Let $i: Z_{0} \rightarrow X$ be the inclusion and denote by $i_{*}$ the composition of the Thom isomorphism $\lambda_{0}$ and the natural map $K_{S^{1}}^{*}\left(\bar{X}_{0}, \partial \bar{X}_{0}\right) \rightarrow K_{S^{1}}^{*}(X)$. Then

$$
i^{*} i_{*}(x)=\lambda_{-1}(v) \cdot x, \text { for } x \in K_{S^{1}}^{*}\left(Z_{0}\right)
$$


and $v$ the $S^{1}$ normal bundle of $Z_{0}$ in $X$. From the exact sequence of the pair $\left(X, \bar{X}_{1}\right)$ we obtain this short exact sequence

$$
0 \rightarrow K_{S^{1}}^{*}\left(Z_{0}\right) \stackrel{i_{*}}{\rightarrow} K_{S^{1}}^{*}(X) \stackrel{j *}{\rightarrow} K_{S^{1}}^{*}\left(Z_{1}\right) \rightarrow 0
$$

where $j: Z_{1} \rightarrow X$ is the inclusion.

Let $\eta_{i} \in K_{S^{1}}^{*}\left(Z_{i}\right)$ be the equivariant Hopf bundle for $Z_{i}$ and $\eta \in K_{S^{1}}^{*}(X)$ the equivariant Hopf bundle for $X$. Since $1, \eta_{i}$ gives an $R\left(S^{1}\right)$ base for $K_{S^{1}}^{*}\left(Z_{i}\right)$, it follows readily from (i) that $i_{*}(1), i_{*}\left(\eta_{0}\right), 1$ and $\eta$ gives an $R\left(S^{1}\right)$ base for $K_{S^{1}}^{*}(X)$. Let

$$
e_{i}=\prod_{j \neq i}\left(\eta-t^{a_{j}}\right)\left(t^{a_{i}}-t^{a_{j}}\right)^{-1} \in K_{S^{1}}^{*}(X) \otimes_{R\left(S^{1}\right)} F\left(S^{1}\right)=K_{S^{1}}^{*}(X)_{0} .
$$

LeMma 5.1. In $K_{S^{1}}^{*}(X)_{0}$ we have

$$
\begin{aligned}
i_{*}(1) & =\lambda_{-1}(v)\left(e_{0}+e_{1}\right), \\
i_{*}\left(\eta_{0}\right) & =\lambda_{-1}(v)\left(e_{0}+t^{7} e_{1}\right), \\
1 & =e_{0}+e_{1}+e_{2}+e_{3}, \\
\eta & =e_{0}+t^{7} e_{1}+t^{1} e_{2}+t^{6} e_{3} .
\end{aligned}
$$

REMARK. Note $\lambda_{-1}(v)=\left(1-t^{2}\right)\left(1-t^{3}\right) \cdot 1,1 \in K_{S^{1}}^{*}(X)$; so we regard $\lambda_{-1}(v)$ as $\left(1-t^{2}\right)\left(1-t^{3}\right) \in R\left(S^{1}\right)$ as well as an element of $K_{S^{1}}^{*}(X)$.

Proof. $K_{S^{1}}^{*}(X)$ is a free $F\left(S^{1}\right)$ module so the restriction

$$
r^{*}: K_{S^{1}}^{*}(X)_{0} \rightarrow K_{S^{1}}^{*}\left(X^{S^{1}}\right)_{0}=\prod_{i=0}^{3} K_{S^{1}}^{*}\left(p_{i}\right)_{0}
$$

is an isomorphism; hence, in order to establish the equation of the lemma it is sufficient to show that they hold in $K_{S^{1}}^{*}\left(X^{S^{1}}\right)_{0}$. E.g., since $j^{*} i_{*}=0$, $i_{*}(1)=\alpha_{0} e_{0}+\alpha_{1} e_{1}$ and $\alpha_{i}=\left.i_{*}(1)\right|_{p_{i}}$ for $i=0,1$. But $\left.i_{*}(1)\right|_{p_{i}}=\left.i^{*} i_{*}(1)\right|_{p_{i}}$ $=\left.\lambda_{-1}(v)\right|_{p_{i}}=\lambda_{-1}(v)$. Thus $\alpha_{0}=\alpha_{1}=\lambda_{-1}(v)$.

THEOREM 5.2. The bilinear form $\left\langle>\right.$ on $K_{S^{1}}^{*}(X)$ is nondegenerate.

PROOF. With respect to the basis $e_{i}$, the matrix of the bilinear form $\langle>$ is diagonal. In fact,

$$
\begin{aligned}
& \left\langle e_{0}, e_{0}\right\rangle=t^{-\lambda_{0}} /\left(1-t^{7}\right) \lambda_{-1}(v), \\
& \left\langle e_{1}, e_{1}\right\rangle=t^{-\lambda_{1}} /\left(1-t^{-7}\right) \lambda_{-1}(v), \\
& \left\langle e_{2}, e_{2}\right\rangle=t^{-\lambda_{2}} /\left(1-t^{5}\right) \lambda_{-1}(v), \\
& \left\langle e_{3}, e_{3}\right\rangle=t^{-\lambda_{3}} /\left(1-t^{-5}\right) \lambda_{-1}(v),
\end{aligned}
$$

where $\lambda_{i}$ are integers. See Part I, Corollary 5.6. 
So in terms of this basis the determinant of $\langle>$ is

$$
D=u /\left(1-t^{7}\right)^{2}\left(1-t^{5}\right)^{2} \cdot \lambda_{-1}(v)^{4}
$$

where $u$ is a unit of $Z\left[t, t^{-1}\right]$.

Let $S$ be the matrix which expresses the basis $i_{*}(1), i_{*}\left(\eta_{0}\right), 1, \eta$ in terms of the basis $\left\{e_{i}\right\}$. From Lemma 5.1 we see that the determinant of $S$, written $|S|$, is $\lambda_{-1}(v)^{2} \cdot\left(1-t^{7}\right)\left(1-t^{5}\right) \cdot t$. Thus the determinant of \langle\rangle with respect to the "integral basis" $i_{*}(1), i_{*}(\eta), 1, \eta$ is $|S|^{2} \cdot D$ and this is a unit of $Z\left[t, t^{-1}\right]$.

I regard this example optimistically as strong evidence that the bilinear form \langle\rangle is nondegenerate under rather general circumstances.

\section{BIBLIOGRAPHY}

0. M. F. Atiyah, On the K-theory of compact Lie groups, Topology 4 (1965), 95-99. MR 31 \# 2350

1. $-K$-theory, Benjamin, New York, 1967. MR 36 \# 7130.

2. - Vector fields on manifolds, Arbeitsgemeinschaft für Forschung des Landes Nordrhein-Westfalen, Heft 200, Westdeutscher Verlag, Cologne, 1970. MR 41 \#7707.

3. M. F. Atiyah, R. Bott and A. Shapiro, Clifford modules, Topology 3 (1964), suppl. 1, 3-38. MR 29 \# 5250.

4. M. F. Atiyah and F. Hirzebruch, Spin-manifolds and group actions, Essays on Topology and Related Topics, Springer-Verlag, New York, 1969, pp. 18-28.

5. M. F. Atiyah and G. Segal, The index of elliptic operators. II, Ann. of Math. (2) 87 (1968), 531-545. MR 38 \# 5244.

6. M. F. Atiyah and I. Singer, The index of elliptic operators. I, III, Ann. of Math. (2) 87 (1968), 484-530, 546-604. MR 38 \# 5243; \# 5245.

7. H. Bass, Algebraic K-theory, Benjamin, New York, 1968. MR 40 \# 2736.

8. R. Bott, The index theorem for homogeneous differential operators, Differential and Combinatorial Topology (A Symposium in Honor of Marston Morse), Princeton Univ. Press, Princeton, N.J., 1965, pp. 167-186. MR 31 \# 6246.

9. G. E. Bredon, The cohomology ring structure of a fixed point set, Ann. of Math. (2) 80 (1964), 524-537. MR 32 \#1698.

10. L. Hodgkin, An equivariant Künneth formula in K-theory, Notes, University of Warwick.

11. W. Y. Hsiang, On generalizations of a theorem of $A$. Borel and their applications in the study of topological actions, Topology of Manifolds, Markham, Chicago, Ill., 1970, pp. 274-290.

12. C. N. Lee, Equivariant homology theories, Proc. Conference on Transformation Groups (New Orleans, La., 1967), Springer, New York, 1968, pp. 237-244. MR 40 \# 3538.

13. J. Milnor, The representation rings of some classical groups, Notes, Princeton University, Princeton, N.J., 1963.

14. Infinite cyclic coverings, Conference on the Topology of Manifolds (Michigan State Univ., E. Lansing, Mich., 1967), Prindle, Weber \& Schmidt, Boston, Mass., 1968, pp. 115-133. MR 39 \#3497.

15. T. E. Stewart, Lifting the action of a group in a fiber bundle, Bull. Amer. Math. Soc. 66 (1960), 129-132. MR 22 \#2994.

16. J. C. Su, Transformation groups on cohomology projective spaces, Trans. Amer. Math. Soc. 106 (1963), 305-318. MR 26 \# 1389.

17. D. Sullivan, Geometric topology seminar, Notes, Princeton University, Princeton, N.J., 1967.

18. A. Vasquez, Poincaré duality for $K_{G}(G / H)$ (to appear).

19. D. Montgomery and C. T. Yang, Free differentiable actions on homotopy seven spheres. II, Proc. Conference on Transformation Groups, (New Orleans, La., 1967), Springer, New York, 1968, pp. 1425-134. MR 39 \#6353.

Department of Mathematics, Rutgers University, New Brunswick, New Jersey 08903 